\title{
High-Precision Multiphoton Ionization of Accelerated Laser-Ablated Species
}

\author{
R. F. Garcia Ruiz, ${ }^{1,2, *}$ A. R. Vernon, ${ }^{1}$ C. L. Binnersley, ${ }^{1}$ B. K. Sahoo, ${ }^{3}$ M. Bissell, ${ }^{1}$ J. Billowes, ${ }^{1}$ T. E. Cocolios ${ }^{4}$ \\ W. Gins, ${ }^{4}$ R. P. de Groote, ${ }^{4, \dagger}$ K. T. Flanagan, ${ }^{1,5}$ A. Koszorus ${ }^{4}$ K. M. Lynch, ${ }^{2}$ G. Neyens, ${ }^{4,2}$ C. M. Ricketts, ${ }^{1}$ \\ K. D. A. Wendt, ${ }^{6}$ S. G. Wilkins, ${ }^{1}$ and X. F. Yang ${ }^{4,7}$ \\ ${ }^{1}$ School of Physics and Astronomy, The University of Manchester, Manchester M13 9PL, United Kingdom \\ ${ }^{2}$ EP Department, CERN, CH-1211 Geneva 23, Switzerland \\ ${ }^{3}$ Atomic and Molecular Physics Division, Physical Research Laboratory, Navrangpura, \\ Ahmedabad 380009, India and State Key Laboratory of Magnetic Resonance and Atomic \\ and Molecular Physics, Wuhan Institute of Physics and Mathematics, \\ Chinese Academy of Sciences, Wuhan 430071, China \\ ${ }^{4}$ KU Leuven, Instituut voor Kern-en Stralingsfysica, B-3001 Leuven, Belgium \\ ${ }^{5}$ Photon Science Institute Alan Turing Building, University of Manchester, \\ Manchester M13 9PY, United Kingdom \\ ${ }^{6}$ Institut für Physik, Johannes Gutenberg-Universität Mainz, D-55128 Mainz, Germany \\ ${ }^{7}$ School of Physics and State Key Laboratory of Nuclear Physics and Technology, \\ Peking University, Beijing 100871, China
}

(Received 9 October 2017; revised manuscript received 7 August 2018; published 8 October 2018)

\begin{abstract}
We demonstrate that the pulsed-time structure and high-peak ion intensity provided by the laser-ablation process can be directly combined with the high resolution, high efficiency, and low background offered by collinear resonance ionization spectroscopy. This simple, versatile, and powerful method offers new and unique opportunities for high-precision studies of atomic and molecular structures, impacting fundamental and applied physics research. We show that even for ion beams possessing a relatively large energy spread, high-resolution hyperfine-structure measurements can be achieved by correcting the observed line shapes with the time-of-flight information of the resonantly ionized ions. This approach offers exceptional advantages for performing precision measurements on beams with large energy spreads and allows measurements of atomic parameters of previously inaccessible electronic states. The potential of this experimental method in multidisciplinary research is illustrated by performing, for the first time, hyperfinestructure measurements of selected states in the naturally occurring isotopes of indium, ${ }^{113,115} \mathrm{In}$. Ab initio atomic-physics calculations have been performed to highlight the importance of our findings in the development of state-of-the-art atomic many-body methods, nuclear structure, and fundamental-physics studies.
\end{abstract}

DOI: 10.1103/PhysRevX.8.041005

\author{
Subject Areas: Atomic and Molecular Physics, \\ Interdisciplinary Physics, \\ Nuclear Physics
}

\section{INTRODUCTION}

The experimental and theoretical study of atomic and molecular structures allows the exploration of a wide range of physical phenomena [1]. In the atom, for example, a precise knowledge of the interaction between the atomic

\footnotetext{
*ronald.fernando.garcia.ruiz@cern.ch

†Present address: Department of Physics, University of Jyväskylä, Jyväskylä, Finland.

Published by the American Physical Society under the terms of the Creative Commons Attribution 4.0 International license. Further distribution of this work must maintain attribution to the author(s) and the published article's title, journal citation, and DOI.
}

nucleus and the surrounding electrons allows subtle details of the electroweak interaction to be studied and an exploration of the possible violation of fundamental symmetries [2,3]. Moreover, atomic hyperfine-structure measurements provide access to nuclear electromagnetic properties that are key for our understanding of the nuclear many-body problem [4,5]. Alongside the experimental progress, the development of many-body methods plays a central role as it provides the means to extract nuclear structure and fundamental physics observables from experimental measurements [6-8].

From the experimental point of view, the precision frontier aims to provide a deeper knowledge of the underlying electron-nucleon interactions and to probe the existence of new physics beyond the standard model $[3,9,10]$. 
On the other hand, the quest to explore the evolution of nuclear properties at the limits of stability has motivated the development of highly sensitivity techniques [11-13]. Although these experimental developments in precision and sensitivity impact different research fields, their direct applications in other disciplines are, in many cases, limited by the complexity of the experimental techniques. In this work, we present a simple yet powerful experimental approach that can be used to perform highly sensitive and precise measurements of atomic- and molecular-structure observables. The approach combines distinct features provided by pulsed-laser ablation [14-16], with the recent developments of collinear resonance ionization spectroscopy (CRIS) at ISOLDE-CERN [11,17]. The latter technique has been developed to perform hyperfine-structure measurements of short-lived radioactive isotopes using multiple laser fields to stepwise excite and ionize accelerated atoms. As the removal of an electron into the atomic continuum is typically a nonresonant process, high-power pulsed lasers are required for efficient ionization. These are commonly only available with low repetition rates $(200 \mathrm{~Hz})$. This has been a major limitation in the application of the collinear resonance ionization approach, as duty-cycle losses caused by the use of pulsed lasers with continuous ion beams dramatically reduce the method's efficiency [18]. Hence, the use of ion traps to bunch the ion beam in synchronization with the laser pulses has been crucial for the high efficiency of the CRIS technique $[11,19]$. Thanks to advancements in ion traps, the high efficiency of the CRIS technique has been achieved [11], more than two decades after its initial demonstration [18].

In this work, we demonstrate that the pulsed-time structure of the ablation process can be used to avoid the technical complexity of using ion traps. Moreover, an inherent time-of-flight (TOF) mass separation provided by the use of accelerated ion beams, in addition to the kinematic shift of the atomic transitions and the high selectivity of the resonance ionization technique, allows ultralow-background measurements to be performed, without the need of mass-separation devices. We show that a significant enhancement in precision can be achieved by using the time-of-flight information of the laser-ionized ions. Such advantages provided by accelerated laserablated ions considerably simplify the CRIS approach and pave the way for applications beyond radioactive beam facilities.

The feasibility of the experimental scheme is demonstrated by performing, for the first time, isotope-shift and hyperfinestructure measurements of the 246.0-nm $\left(5 p^{2} P_{1 / 2} \rightarrow\right.$ $\left.8 s^{2} S_{1 / 2}\right)$, 246.8-nm $\left(5 p^{2} P_{3 / 2} \rightarrow 9 s^{2} S_{1 / 2}\right)$, and 283.7-nm $\left(5 p^{2} P_{3 / 2} \rightarrow 5 s 5 p^{2}{ }^{4} P_{5 / 2}\right)$ transitions in the naturally occurring indium isotopes, ${ }^{113,115} \mathrm{In}$. We have chosen atomic indium (In) to illustrate the impact of our findings in multidisciplinary research, as it exhibits distinct properties that are of interest in nuclear structure, atomic physics, and studies of fundamental symmetries. (i) It has been proposed as a candidate for electric dipole moment (EDM) measurements [20]. Furthermore, its properties constitute a testing ground for atomic-physics calculations directly applicable to other elements of interest in fundamental-physics research, e.g., thallium ( $\mathrm{Tl}$ ), rubidium ( $\mathrm{Rb}$ ), and cesium (Cs) [21-25]. High-precision measurements of parity nonconservation (PNC) [26,27] and EDM [28] have been performed in the $\mathrm{Tl}$ atom, which is a homologous three valence-electron system from the same periodic group as In. However, conclusive findings from high-precision experiments in these atomic systems are limited by the accuracy of the theoretical calculations needed to disentangle fundamental-physics parameters from atomic observables $[22,23,26,29]$. (ii) The matrix elements of weak-interaction Hamiltonians involved in the electron-nucleon interaction are not experimental observables; quantifying the uncertainty of their theoretical predictions is therefore of paramount importance. Since these matrix elements, as well as the hyperfine interactions, originate from the interaction between the electrons and the atomic nucleus, PNC and EDM calculations are usually adjudged by comparing calculated magnetic dipole hyperfine-structure constants with available measurements [20-22]. In this work, we have measured, for the first time, the hfs constants of atomic states in In that are relevant in these studies. (iii) Because of the large interest in the indium atom, high-precision values are available in literature for several atomic states. These high-precision data provide an excellent case to benchmark the results of our experimental method. (iv) With a single proton hole in a nuclear closed shell, the indium isotopes $(Z=49)$ are of marked importance for nuclear structure studies [30,31]. We have studied the sensitivity of different atomic states to nuclear structure observables, and their feasibility for future studies with short-lived isotopes. This information is crucial for the success of a current experimental program approved at CERN on the study of rare indium isotopes [32]. (v) Presently, the uncertainties of the nuclear quadrupole moments and nuclear charge radii of the indium isotopes are limited by the accuracy of the atomic many-body methods used to extract nuclear structure parameters from hyperfinestructure measurements. Testing the reliability of the available atomic many-body methods is critical to assess the accuracy of the atomic calculations used to extract nuclear structure information. The importance of our results in the development of atomic-physics theory and their relevance for studying fundamental and nuclear structure physics is highlighted through the comparison with ab initio atomic-physics calculations.

\section{EXPERIMENTAL SETUP}

\section{A. Ion-source setup}

An ion-source setup was developed to perform collinear laser spectroscopy experiments on stable ions generated by 


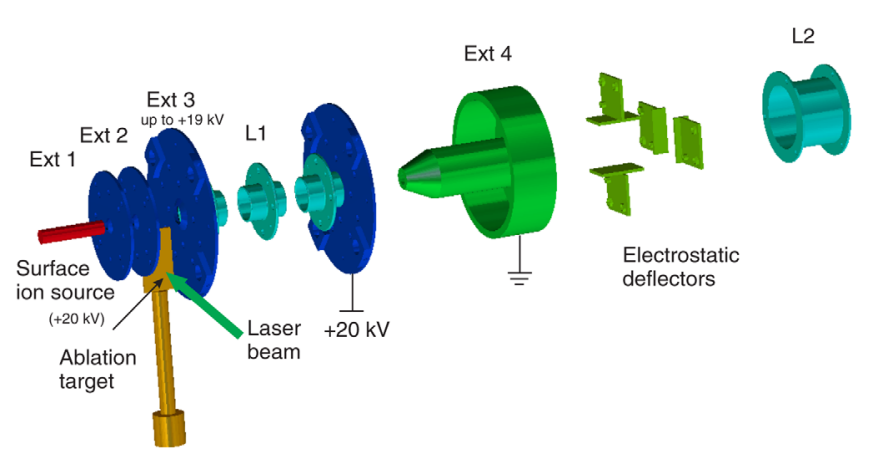

FIG. 1. Ion-source setup. Schematic of the ion-source setup used for the study of laser-ablated ions. See text for details of the electrostatic elements.

ablation and surface ionization. A schematic of this setup is shown in Fig. 1. A simple ion extraction system allows a fast exchange between the two ion-source types without requiring modification of the ion-beam optics. A hot-capillary tube is used for surface ionization. Alternatively, a pulsed-laser beam perpendicular to the ion-beam axis can be used to ablate material from solid targets. Different materials can be selected from a sample holder installed on an adjustable linear feedthrough.

The surface ion source can produce relatively pure continuous ion beams with an energy spread of about $1 \mathrm{eV}$. However, surface ionization is limited to elements with low ionization potentials [33]. The ablation ion source can be used to produce ions of a wide range of elements that are not accessible by surface ionization [34]. Furthermore, ions ablated by pulsed lasers exhibit unique features such as a pulsed time structure and a high peak intensity. These characteristics are essential to performing high-efficiency collinear resonance ionization spectroscopy, as it guarantees an ideal temporal and spacial laser-atom overlap. However, the complexity of the ablation process [35-37] has the potential to create a large range of energy spreads, which could impose a major disadvantage for using ablation sources in precision studies. Our results directly address these issues, demonstrating that high-precision measurements on laser-ablated ions can be achieved, even for ion beams with a large energy spread.

The indium isotopes studied in this work were ionized by laser ablation from 532-nm laser pulses focused on a solid indium target with a purity of $99 \%$. The ablated ions were accelerated to $20 \mathrm{keV}$ using a multiple-step extraction system (see Fig. 1). An electrode maintained at a negative potential (Ext 3) with respect to the ion-source potential $(+20 \mathrm{kV})$ was used to extract the ablated ions. The extracted ions were then refocused and redirected into an extractor electrode located at ground potential (Ext 4), accelerating the ion beam up to the final energy $(20 \mathrm{keV})$. A set of horizontal- and vertical-electrostatic plates were used to direct the ion beam into the center of an einzel lens (L2). The latter is used to correct the focusing effect caused by the high extraction voltage. Ablated ions were produced with several charge states. However, the electrostatic deflectors along the beam path serve as energy selectors, and the time-of-flight information of the ion bunch can be used to select the singly charged indium ions of interest.

\section{B. Laser system}

An injection-seeded titanium-sapphire (Ti:sapphire) laser system was used to provide the narrow-band laser pulses $(\sim 50 \mathrm{MHz})[38,39]$. Seeded by continuous-wave light provided by an M-Squared SolsTiS Ti:sapphire laser, the injection-seeded cavity was pumped by a $Q$-switched $\mathrm{Nd}$ :YAG laser (Lee Laser LDP-100MQG) at a repetition rate of $1 \mathrm{kHz}$. The required wavelengths for the resonance transitions (246.0, 246.8, and $283.7 \mathrm{~nm}$ in Fig. 2) were produced by frequency tripling the injection-seeded light with two nonlinear crystals. The 410.2-nm light was produced by frequency doubling the injection-seeded light with one nonlinear crystal. The laser frequencies were scanned by tuning the M-Squared wavelength, and measured with a WSU-2 HighFinesse wavelength meter. The wavelength meter was calibrated by measuring a reference wavelength provided by a stabilized diode laser (Toptica DLC DL PRO 780), which was locked to an atomic transition in rubidium. The resonant step corresponding to the 571.0-nm transition was obtained from a Spectron PDL SL4000 laser, pumped by a high-energy Nd:YAG laser (Litron TRLi 250-100) $Q$ switched at a repetition rate of $100 \mathrm{~Hz}$. The 1064- and 532-nm nonresonant ionization steps were provided by a high-energy Nd:YAG laser (Litron LPY 601 50-100 PIV) $Q$ switched at a repetition rate of $100 \mathrm{~Hz}$. The laser beam waist along the laser-atom interaction region was approximately $8 \mathrm{~mm}$, with a fluence of 120 and $60 \mathrm{~mJ} / \mathrm{cm}^{2}$ for 1064 and $532 \mathrm{~nm}$, respectively. An independent head of the Nd:YAG laser provided 532-nm pulsed laser light used for the ablation ion source. These laser pulses were focused into the ablation target with a

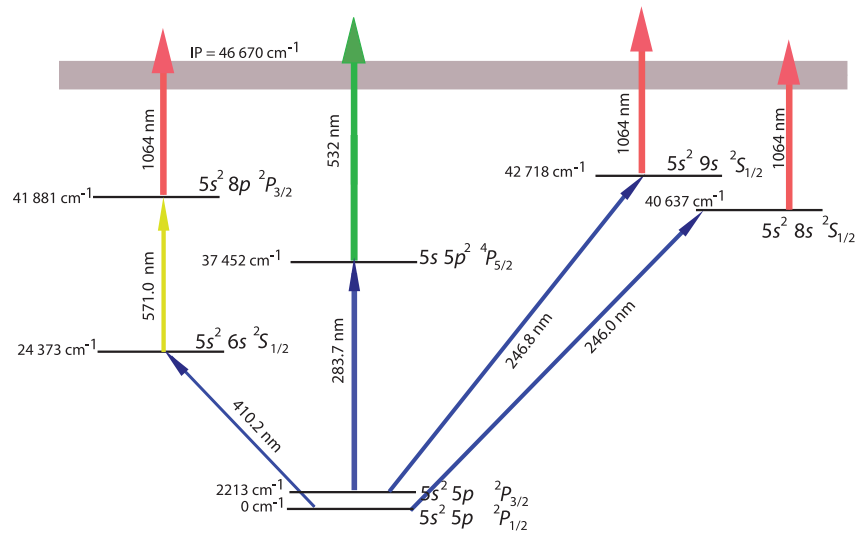

FIG. 2. Resonance-ionization schemes in the indium atom. The 410.2-, 283.7-, 246.8-, and 246.0-nm resonant transitions that were studied in this work. 
laser beam waist of approximately $1 \mathrm{~mm}$, corresponding to a fluence of $0.5 \mathrm{~J} / \mathrm{cm}^{2}$. A Quantum Composers 9528 digital delay pulse generator was used to synchronize the laser pulses with the ablated ions.

\section{Collinear laser spectroscopy}

The acceleration of the ions to the final energy $E$ significantly reduces the velocity spread of the ion beam. As the initial energy spread $\delta E$ is conserved through acceleration, the velocity spread of the accelerated beam is reduced by $\delta \nu=\delta E / \sqrt{2 m E}$, where $m$ is the mass of the ion. This reduction of Doppler broadening and the optimal overlap between the atoms and the laser beams are some of the main advantages of performing laser spectroscopy on accelerated beams for high-resolution studies [40,41]. An energy spread of $10 \mathrm{eV}$, for example, causes a Doppler broadening of $27 \mathrm{GHz}$ at wavelengths around $246 \mathrm{~nm}$. This large Doppler broadening does not allow individual transitions between different hyperfine-structure levels to be resolved, which are typically separated by less than $1 \mathrm{GHz}$. However, by accelerating the ions up to $20 \mathrm{keV}$, the velocity spread decreases significantly, reducing the Doppler broadening between 100 and $400 \mathrm{MHz}$. This velocity-spread reduction of nearly 2 orders of magnitude allowed a complete separation of the hfs peaks for the In atomic transitions studied. As we explain in Sec. IVA, further reduction of the Doppler broadening down to $50 \mathrm{MHz}$ was achieved through correction of the resonant line shape using the time-of-flight information.

After the $20-\mathrm{kV}$ acceleration, the bunches of laserablated ions were redirected into the CRIS experiment at
ISOLDE-CERN [11,17]. A schematic of the experimental setup is shown in Fig. 3. As the states of interest are in atomic indium, the ions were neutralized in flight by using a sodium-vapor cell at approximately $250{ }^{\circ} \mathrm{C}$. After the neutralization process, the non-neutralized ions are removed by an electrostatic deflector. The neutral atoms follow a straight path towards an interaction region held at $3 \times 10^{-10}$ mbar, where they are collinearly overlapped with the laser pulses. In this region, a narrow-band laser pulse is used to resonantly excite the indium atoms to the atomic state of interest. The use of pulsed lasers provides significant advantages for performing resonance ionization. The high-peak photon densities produced by pulsed lasers allow transitions to be easily saturated, allowing efficient resonance ionization to occur. This high peak-photon density means that production of deep-UV light can be achieved with relatively simple setups when compared to the expensive and complex setups required for efficient higher-harmonic generation of continuous-wave light. A second high-power laser pulse (532 or $1064 \mathrm{~nm}$ ) is used to promote the electron from the excited state into the atomic continuum. The resonantly ionized ions are then separated from the noninteracting atoms via electrostatic deflector plates, and are detected by a microchannel plate (MCP) particle detector. The background associated with the dark count rate of the MCP and collisional ionization of beam contaminants is highly suppressed by setting a $4-\mu$ s gate on the signals, corresponding to the time of flight of the atom bunch of interest along the interaction region.

The insets in Fig. 3 show the simulated hfs spectra for ${ }^{113}$ In and ${ }^{115}$ In in the 246.8-nm transition. At rest, the hfs



FIG. 3. Experimental setup used for the study of indium isotopes. Bunches of laser-ablated indium ions were accelerated up to an energy of $20 \mathrm{keV}$ and neutralized in flight by a neutralization cell loaded with sodium. Remaining ions were removed by an electrostatic deflector, and the neutralized atoms followed a straight path towards an interaction region, where they were collinearly overlapped with the laser beams. The hyperfine-structure spectra were measured by recording the count rate on the MCP detector as a function of the resonant laser frequency. The insets show the simulated hfs spectra for ${ }^{113} \mathrm{In}$ and ${ }^{115} \mathrm{In}$ using the 246.8 -nm transition. The spectra are compared with the Doppler-broadened spectrum (red color) and Doppler-free spectra for ions at rest and accelerated up to $20 \mathrm{keV}$. 
spectra of these two isotopes exhibits a large overlap. Therefore, they are particularly difficult to separate with conventional resonance ionization mass spectroscopy techniques (RIMS) such as in-source RIMS (Doppler-broadened hfs spectra) and cross beam RIMS (Doppler-free) [42]. However, for accelerated ions, the kinematic shift at $20 \mathrm{keV}$ provides a clear separation of both hfs spectra.

\section{ATOMIC THEORY AND COUPLED-CLUSTER METHOD}

The first-order change in the energy of an atomic energy level with angular momentum $J$ due to the magnetic dipole $(M 1)$ and electric quadrupole (E2) hyperfine interactions in atomic units (a.u.) is given by [43]

$$
W_{F, J}^{(1)} \simeq W_{F, J}^{M 1}+W_{F, J}^{E 2},
$$

which are conveniently expressed as

$$
W_{F, J}^{M 1}=A_{\mathrm{hf}} \mathbf{I} . \mathbf{J}
$$

and

$$
W_{F, J}^{E 2}=B_{\mathrm{hf}} \frac{3(\mathbf{I} . \mathbf{J})^{2}+\frac{3}{2}(\mathbf{I} . \mathbf{J})-I(I+1) J(J+1)}{2 I(2 I-1) J(2 J-1)} .
$$

The $A_{\mathrm{hf}}$ and $B_{\mathrm{hf}}$ constants are defined as

$$
A_{\mathrm{hf}}=g_{I} \mu_{N} \frac{\left\langle J|| T_{e}^{(1)}|| J\right\rangle}{\sqrt{J(J+1)(2 J+1)}}
$$

and

$$
B_{\mathrm{hf}}=2 Q\left(\frac{J(2 J-1)}{(J+1)(2 J+1)(2 J+3)}\right)^{1 / 2}\left\langle J\left\|T_{e}^{(2)}\right\| J\right\rangle,
$$

respectively. With $\mu_{N}$ the nuclear magneton, $Q$ the spectroscopic nuclear quadrupole moment, and the gyromatic factor $g_{I}=\mu_{I} / I$, defined as the ratio between the nuclear magnetic moment $\mu_{I}$ (in units of nuclear magnetons) and nuclear spin $I$. In the above expressions, the operators

$$
T_{e}^{(1)}=\sum_{j, q} t_{q}^{(1)}\left(r_{j}\right)=\sum_{j, q}-i e \sqrt{8 \pi / 3} r_{j}^{-2} \alpha_{j} . \mathbf{Y}_{\mathbf{1 q}}^{(\mathbf{0})}\left(\mathbf{r}_{\mathbf{j}}\right)
$$

and

$$
T_{e}^{(2)}=\sum_{j, q} t_{q}^{(2)}\left(r_{j}\right)=\sum_{j, q}-i e r_{j}^{-3} C_{q}^{(2)}\left(r_{j}\right)
$$

are the electronic components of hyperfine interactions, whose single-particle matrix elements are given by

$$
\begin{aligned}
\left\langle\kappa m\left|t_{q}^{(1)}\right| \kappa^{\prime} m^{\prime}\right\rangle= & -\left\langle-\kappa m\left|C_{q}^{(1)}\right| \kappa^{\prime} m^{\prime}\right\rangle\left(\kappa+\kappa^{\prime}\right) \\
& \times \int_{0}^{\infty} d r \frac{\left(P_{\kappa} Q_{\kappa^{\prime}}+P_{\kappa} Q_{\kappa^{\prime}}\right)}{r^{2}}
\end{aligned}
$$

and

$$
\begin{aligned}
\left\langle\kappa m\left|t_{q}^{(2)}\right| \kappa^{\prime} m^{\prime}\right\rangle= & -\left\langle\kappa m\left|C_{q}^{(2)}\right| \kappa^{\prime} m^{\prime}\right\rangle \\
& \times \int_{0}^{\infty} d r \frac{\left(P_{\kappa} P_{\kappa^{\prime}}+Q_{\kappa} Q_{\kappa^{\prime}}\right)}{r^{3}},
\end{aligned}
$$

where $\kappa_{i}$ is the relativistic angular-momentum quantum number of the $i$ th Dirac orbital with the large radial component $P_{i}$ and the small radial component $Q_{i}$.

To obtain the single-particle orbitals, the Dirac-HartreeFock (DHF) approach is considered for calculating the mean-field wave function $\left(\left|\Phi_{0}\right\rangle\right)$ of the closed-shell configuration $\left[5 s^{2}\right]$ of $\mathrm{In}^{+}$. For this purpose, the Dirac-Coulomb (DC) atomic Hamiltonian is used, accounting for corrections due to the Breit and quantum electrodynamics (QED) interactions [44]. For the initial construction of the orbitals for DHF wave functions, analytical basis functions having quadratic-type exponents are used, labeled as quadratic-type orbitals (QTOs). After obtaining $\left|\Phi_{0}\right\rangle$ with different levels of the approximations in the Hamiltonian, the mean-field wave function of the ground and excited states of In with the closed-shell configuration $\left[5 s^{2}\right]$ and a corresponding valence orbital $v$ is defined as $\left|\Phi_{v}\right\rangle=a_{v}^{\dagger}\left|\Phi_{0}\right\rangle$. These approximated valence orbitals are the virtual orbitals of the DHF wave function of the closed-shell configuration. To account for the electron-correlation effects due to the residual Coulomb (and Breit) interactions in the DHF method, a relativistic coupled-cluster (RCC) approach is used to determine the atomic wave functions. In the RCC ansatz, wave functions of an atomic state with a closed core and a valence orbital are expressed as [20,45-49]

$$
\left|\Psi_{v}\right\rangle=e^{T}\left\{1+S_{v}\right\}\left|\Phi_{v}\right\rangle
$$

where $T$ and $S_{v}$ are the excitation operators carrying out the core-core and core-valence electron-correlation effects, respectively. By defining the normal order Hamiltonian with respect to the reference state $\left|\Phi_{0}\right\rangle$ and for the electron affinity $\left(\Delta E_{v}\right)$ energy for the valence electron $v$ from $\operatorname{~In}^{+}$, the amplitudes of the $T$ and $S_{v}$ RCC excitation operators are obtained by solving the following equations:

$$
\left\langle\Phi_{0}^{*}\left|\bar{H}_{N}\right| \Phi_{0}\right\rangle=0
$$

and

$$
\left\langle\Phi_{v}^{*}\left|\left(\bar{H}_{N}-\Delta E_{v}\right) S_{v}\right| \Phi_{v}\right\rangle=-\left\langle\Phi_{0}^{*}\left|\bar{H}_{N}\right| \Phi_{v}\right\rangle,
$$

with $\bar{H} \equiv e^{-T} H e^{T}$. The superscript $*$ over the reference states indicates that they refer to the excited determinants 
with respect to the reference states. The subscript $N$ specifies that the operators are in the normal-order form. In our $a b$ initio approach, the $\Delta E_{v}$ value is obtained by

$$
\Delta E_{v}=\left\langle\Phi_{v}\left|\bar{H}_{N}\left\{1+S_{v}\right\}\right| \Phi_{v}\right\rangle .
$$

Since Eqs. (12) and (13) are interdependent, they are solved simultaneously after obtaining the solutions from Eq. (11). We consider all possible singles and doubles excitations through the RCC operators [referred to as the coupledcluster singles doubles method (CCSD)] by defining

$$
T \simeq T_{1}+T_{2} \quad \text { and } \quad S_{v} \simeq S_{1 v}+S_{2 v} .
$$

In our CCSD method, the $T_{1}$ and $S_{2 v}$ operators generate onehole one-particle excitations independently from the reference state $\left|\Phi_{v}\right\rangle$. When these operators act together on the $\left[4 d^{10}\right] 5 s$ and $\left[4 d^{10}\right] 5 p_{1 / 2,3 / 2}$ configurations in the nonlinear form, they also generate the $4 d^{9} 5 s^{2}$ and $4 d^{9} 5 s 5 p$ configurations, respectively. Though the CCSD method accounts for higher-level excitations than the singly and doubly excited determinants through the nonlinear terms, it still misses out on contributions from some of the important lower-order triply excited determinants. Exact inclusion of these excitations is computationally very expensive. To account for lower-order contributions from them, we define the perturbative core and valence triple excitation operators as

$$
T_{3}^{\text {pert }}=\frac{1}{3 !} \sum_{a b c, p q r} \frac{H_{N} T_{2}}{\epsilon_{a}+\epsilon_{b}+\epsilon_{c}-\epsilon_{p}-\epsilon_{q}-\epsilon_{r}}
$$

and

$$
S_{3 v}^{\mathrm{pert}}=\frac{1}{4} \sum_{a b, p q r} \frac{H_{N} T_{2}+H_{N} S_{2 v}}{\epsilon_{v}+\epsilon_{a}+\epsilon_{b}-\epsilon_{p}-\epsilon_{q}-\epsilon_{r}}
$$

where $a, b$ and $p, q, r$ indices represent the occupied and unoccupied orbitals, respectively, and $\epsilon_{s}$ are their corresponding DHF orbital energies. We include these operators in the amplitude and energy solving equations of the CCSD method.

Using the amplitudes of the RCC operators, the expectation values of the respective hyperfine interaction operator $O \equiv T_{e}^{(1)}$ and $O \equiv T_{e}^{(2)}$ for a given state $\left|\Psi_{v}\right\rangle$ are determined by

$$
\langle O\rangle \equiv \frac{\left\langle\Psi_{v}|O| \Psi_{v}\right\rangle}{\left\langle\Psi_{v} \mid \Psi_{v}\right\rangle}=\frac{\left\langle\Phi_{v}\left|\left\{1+S_{v}^{\dagger}\right\} \bar{O}\left\{1+S_{v}\right\}\right| \Phi_{v}\right\rangle}{\left\langle\Phi_{v}\left|\left\{1+S_{v}^{\dagger}\right\} \overline{\mathcal{N}}\left\{1+S_{v}\right\}\right| \Phi_{v}\right\rangle},
$$

where $\bar{O}=e^{T^{\dagger}} O e^{T}$ and $\overline{\mathcal{N}}=e^{T^{\dagger}} e^{T}$ are two nontruncative series appearing in the above expression. These terms are evaluated in iterative procedures, as discussed in our recent works [44-46]. We also estimate contributions from the perturbed triple excitation operators by substituting them in the property evaluating expression as
$\Delta\langle O\rangle=2 \frac{\left\langle\Phi_{v}\left|\left\{T_{2}^{\dagger}+S_{2 v}^{\dagger}\right\} \bar{O}_{o b}\left\{T_{3}^{\text {pert }}+S_{3 v}^{\text {pert }}\right\}\right| \Phi_{v}\right\rangle}{\left\langle\Phi_{v}\left|\left\{1+S_{v}^{\dagger}\right\} \overline{\mathcal{N}}\left\{1+S_{v}\right\}\right| \Phi_{v}\right\rangle}$,

with $\bar{O}_{o b}$ the effective one-body terms of $\bar{O}$. The differences between the results from the CCSD method and after incorporating the triple excitation operators in the above expressions are quoted as the estimated contributions from the "triples" excitations. In this work, the singles, doubles, and partial triples excitation approximation RCC theory is denoted as the $\operatorname{CCSD}(\mathrm{T})$ method. To estimate the uncertainties, we have assumed the triples contributions as the maximum errors due to the neglected higher-level excitations and possible errors due to use of the finite-size basis functions. The latter contributions are obtained by analyzing results using a lower-order many-body method.

\section{RESULTS}

The resonance ionization schemes used in this work are shown in Fig. 2. Four different resonant transitions were studied during the experiment: $246.0 \mathrm{~nm}\left(5 p^{2} P_{1 / 2} \rightarrow\right.$ $\left.8 s^{2} S_{1 / 2}\right), \quad 246.8 \mathrm{~nm} \quad\left(5 p^{2} P_{3 / 2} \rightarrow 9 s^{2} S_{1 / 2}\right), \quad 283.7 \mathrm{~nm}$ $\left(5 p^{2} P_{3 / 2} \rightarrow 5 s 5 p^{2}{ }^{4} P_{5 / 2}\right)$, and $410.2 \mathrm{~nm} \quad\left(5 p^{2} P_{1 / 2} \rightarrow\right.$ $\left.6 s^{2} S_{1 / 2}\right)$. The hfs spectra corresponding to the transitions between different hyperfine states were measured by recording the ion count rate on the MCP detector as a function of narrow-band laser frequency. Examples of the hfs spectra measured for two different extraction field configurations around the ablation plume are shown in Fig. 4. For the larger extraction potential, an extraction field of $5 \mathrm{~V} / \mathrm{mm}$ around the ablation plume was calculated from simulations using the software package COMSOL [50] [Fig. 4(a1)]. The measured time-of-flight distribution exhibited a tail of slower ions [Fig. 4(a2)], and pronounced asymmetric shapes were observed in the hfs spectrum, with a FWHM of 400(50) MHz [Fig. 4(a3)]. For "zero" extraction field, the calculated extraction field was $0.02 \mathrm{~V} / \mathrm{mm}$, associated with the penetrating field from the large potential difference between the ion-source potential $(+20 \mathrm{kV})$ and ground potential [Fig. 4(b1). In this configuration, the tail of the TOF distribution was significantly reduced [Fig. 4(b2)], as well as the asymmetry in the observed hfs spectrum [Fig. 4(b3)]. FWHM values between 80 and $120 \mathrm{MHz}$ were obtained for this extraction potential. All electrostatic elements were optimized for the zero extraction field setting, and the same values were used when the extraction field was applied. In the latter configuration, the integrated intensity of the ions detected on the MCP increased by about $30 \%$. However, this value could potentially be improved with additional beam diagnostic elements that could allow a fast optimization of the ion beam optics for each extraction potential.

The asymmetric line shapes in the measured spectra are attributed to the velocity distribution of the ions generated in the ablation process and the extraction geometry of the 

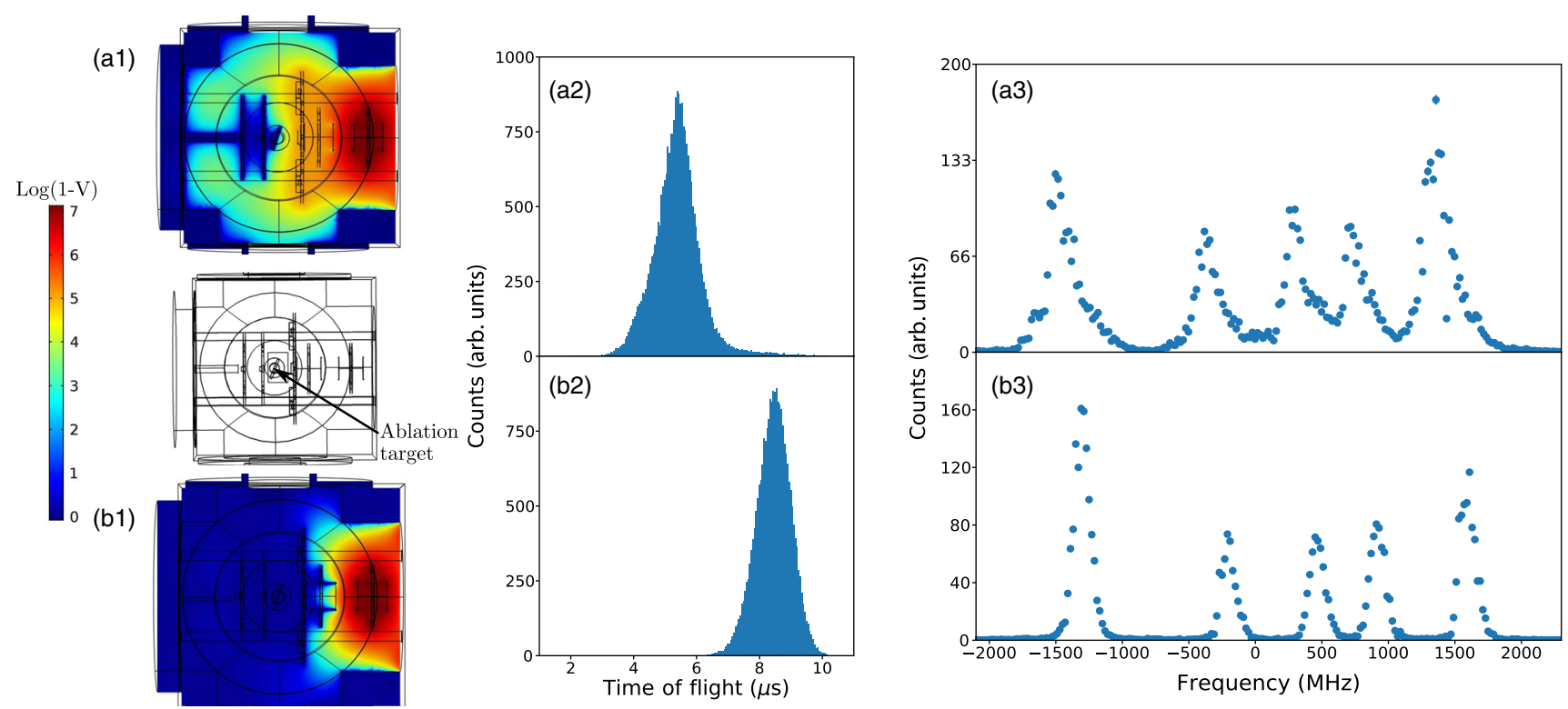

FIG. 4. Influence of extraction potential around the ablation plume. Time-of-flight measurements of laser-ablated ions arriving at the MCP detector (a2),(b2). The measured hyperfine-structure spectra for the 246.8-nm transition of ${ }^{115}$ In (a3), (b3) were obtained for two different extraction potentials (a1),(b1). Panels (a1) and (b1) show the simulations for the corresponding extraction potentials with respect to the ion-source potential. The simulated extraction fields around the ablation plume were, respectively, $5 \mathrm{~V} / \mathrm{mm}(\mathrm{a} 1)$ and $0.02 \mathrm{~V} / \mathrm{mm}(\mathrm{b} 1)$.

source. The ion velocity distribution at the source can be described by a Maxwell-like distribution that takes into account the temperature distribution of ablated ions, and a long tail extended towards higher energies due to additional laser-generated plasma effects [51-53]. The asymmetry of the line shape was found to increase with the extraction field. Depending on the extraction potential, FWHM values between 80 and $400 \mathrm{MHz}$ were obtained for the measured $\mathrm{hfs}$ spectra. This value reflects the temperature of the lasergenerated plasma in the ablation process and the extraction field around the ablation plume. As presented in the following section (Sec. IVA), higher-precision measurements with symmetric line shapes and FWHMs of about $50 \mathrm{MHz}$ were achieved by correcting the hfs spectra with the TOF information of individual ions.

\section{A. Time-of-flight corrected line shapes}

The count rate of resonant ions detected on the MCP and the corresponding excitation laser frequency were recorded with a time stamp of 500-ps time resolution. An example of the observed correlation for these two parameters, during the hyperfine-structure scan for the 246.8-nm line of ${ }^{115} \mathrm{In}$, is shown in Fig. 5. The surface plot shows the counts on the $\mathrm{MCP}$ detector as a function of the laser frequency and TOF of the ions arriving on the MCP detector. The figure shows the measurements for a large extraction voltage, with an extraction field of $5 \mathrm{~V} / \mathrm{mm}$ [Fig. 4(a1)]. The shape of these distributions was found to depend on the extraction voltages applied to the elements around the ablation target. At the top of Fig. 5, the dark orange histogram shows the total hfs spectrum, which exhibits a pronounced asymmetric line shape with FWHM values between 300 and $400 \mathrm{MHz}$. The light orange histograms in Fig. 5 show examples of the obtained hfs spectrum for different TOF

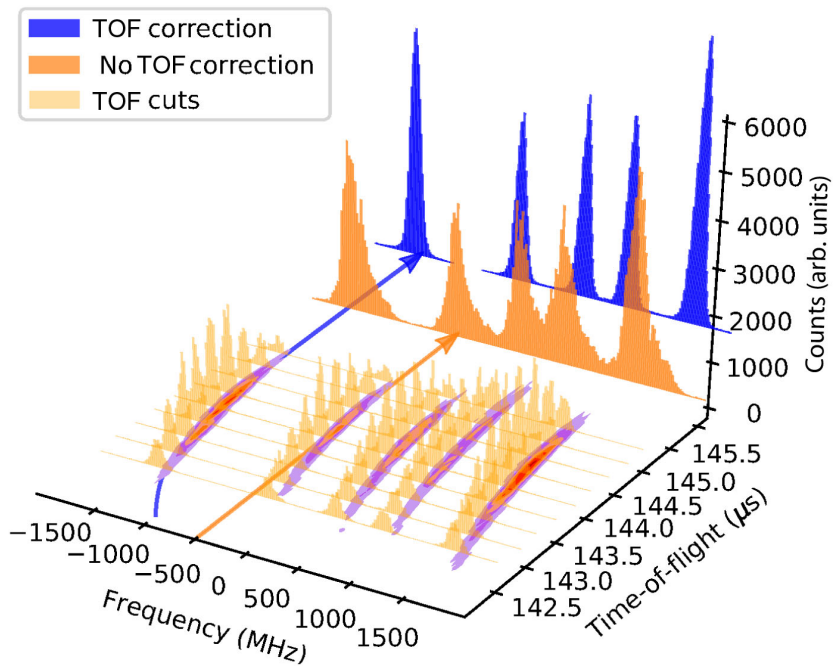

FIG. 5. Time of flight versus laser frequency. Measured hfs spectra for ${ }^{115} \mathrm{In}$ in the 246.8 -nm line using an extraction field of $5 \mathrm{~V} / \mathrm{mm}$ around the ablation plume. The surface plot shows the counts on the MCP detector as a function of the laser frequency ( $x$ axis) and TOF of the ions arriving on the MCP detector ( $y$ axis). Frequency values are relative to the centroid of ${ }^{115} \mathrm{In}$. Examples of hfs spectra obtained for different TOF cuts are shown in light orange. The total hfs spectrum without TOF correction (orange) is compared with the total TOF-corrected spectrum (blue). 
cuts. Such TOF cuts allowed symmetric line profile with FWHM values below $100 \mathrm{MHz}$ to be obtained. This TOF selection is equivalent to selecting a group of ions with reduced energy spread. When the same procedure is applied to the measurements performed with zero extraction potential $(0.02 \mathrm{~V} / \mathrm{mm}$ around the ablation plume $)$, perfectly symmetric line shapes with FWHM values down to $50 \mathrm{MHz}$ were obtained for the extracted hfs spectra. This resolution is limited by the linewidth of the laser system used during the experiments, and could potentially be improved [54].

The measured laser frequencies and TOF of the ions were found to follow a simple correlation (Fig. 5). To obtain this correlation, the total TOF distribution was divided in TOF cuts of $100 \mathrm{~ns}$ each, and the hfs spectrum corresponding to each TOF cut was fitted independently. The fitted hfs centroids were used to derive a corresponding frequency versus TOF dependence. The peak position of each hfs spectrum was frequency shifted by the fitted frequency versus TOF relation, and the total TOF-corrected hfs spectrum was obtained as the sum of the individual frequency-shifted hfs spectra. The blue histogram in Fig. 5 shows total TOF-corrected hfs spectrum. Examples of TOF-corrected hfs spectra measured for each transition using zero extraction field are shown in Fig. 6.

For the 246.0- and 246.8-nm transitions, a complete hfs scan (Fig. 6) was measured in approximately $5 \mathrm{~min}$ at a scanning speed of $15 \mathrm{MHz} / \mathrm{s}$. Because of the large hyperfine splitting of the 410.2-nm $(\sim 30 \mathrm{GHz})$ and $283.7-\mathrm{nm}$ $(\sim 100 \mathrm{GHz})$ transitions, approximately $20 \mathrm{~min}$ and $1 \mathrm{~h}$ were required, respectively, to complete the hfs scans for these transitions. An estimated $10^{5}-10^{6}$ ablated ions/s arrived in the interaction region. For ${ }^{115} \mathrm{In}$, average rates of 500 ions/s were observed from the resonance peaks in the measured hfs spectra, giving an overall efficiency higher than $0.05 \%$. This total efficiency includes the neutralization fraction, ion-beam transport, ionization, and particledetection efficiency. The ion-beam optics at the CRIS beam line were optimized for accepting ion bunches of low emittance from a radio-frequency ion trap installed at the ISOLDE facility. A large fraction of efficiency loss due to the ion-beam transport from the ablation to the MCP detector (about $6 \mathrm{~m}$ ) was expected. Therefore, it could be further improved in a dedicated setup.

Similar to the TOF cut employed to select the hfs spectra, a frequency gate can be applied to select TOF events. As the hfs spectra of the two indium isotopes are clearly resolved, a frequency gate can be used to individually study the TOF distribution of each isotope. The frequencygated TOF distributions of both isotopes are shown on the right-hand side of Fig. 7. As seen from the figure, the TOF distributions of ${ }^{113}$ In and ${ }^{115}$ In overlap in time, and due to the large difference in natural abundance, such a mass separation would not be possible without a frequency selection.
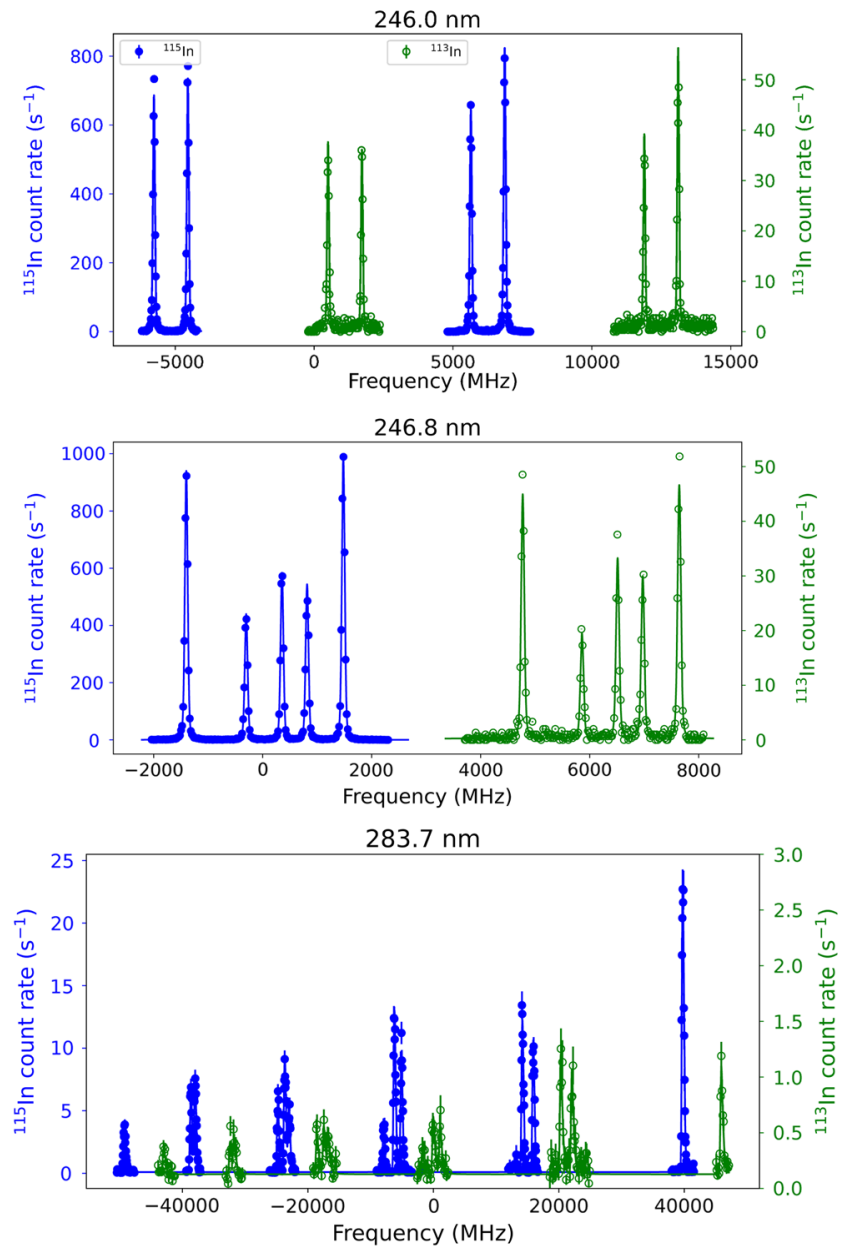

$410.2 \mathrm{~nm}$

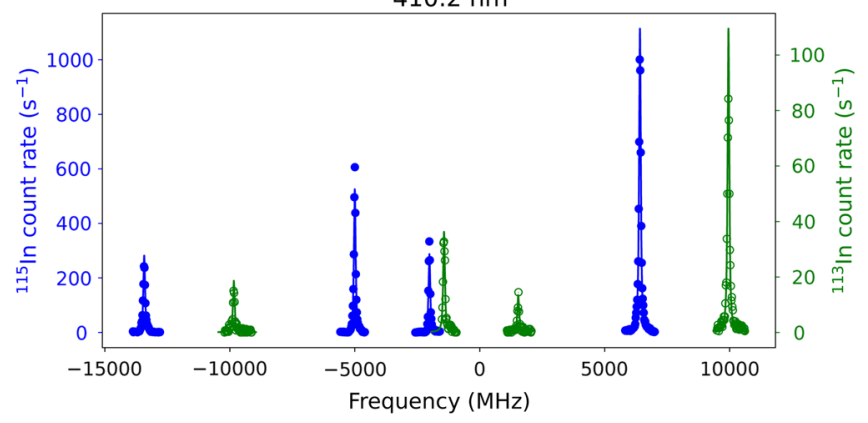

FIG. 6. Measured hyperfine-structure spectra. Examples of TOF-corrected hfs spectra measured for the ${ }^{113}$ In (green) and ${ }^{115}$ In (blue) isotopes in the 246.0-nm (top), 246.8-nm (middle), 283.7-nm (middle), and 410.2-nm (bottom) lines. The continuous lines show the fit with Voigt profiles. Frequency values are shown in the laboratory frame relative to the centroid of ${ }^{115} \mathrm{In}$.

\section{B. Isotope shifts and hyperfine-structure constants}

The experimental magnetic dipole $A_{\mathrm{hf}}$ and electric quadrupole $B_{\text {hf }}$ hfs constants were extracted from the fit of Voigt profiles to the experimental spectra by using a $\chi^{2}$-minimization technique [55]. The differences between the observed peak positions provide the shift of the hyperfine levels $F$ from the unperturbed position (or centroid) [40], 


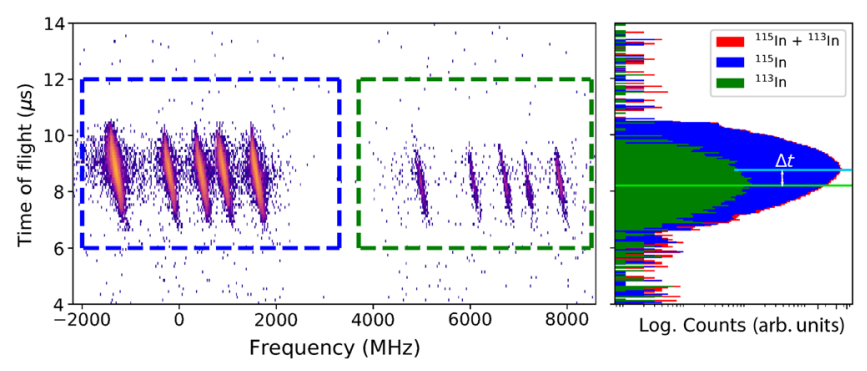

FIG. 7. Frequency cut for isotope selection. Measured hfs spectra for ${ }^{113} \mathrm{In}$ and ${ }^{115} \mathrm{In}$ in the $246.8-\mathrm{nm}$ line using an extraction field of $0.02 \mathrm{~V} / \mathrm{mm}$ around the ablation plume. The surface plot shows the counts on the MCP detector as a function of the laser frequency ( $x$ axis) and TOF of the ions arriving on the MCP detector ( $y$ axis). The blue and green squares show the frequency cuts for the hfs spectra of ${ }^{115} \mathrm{In}$ and ${ }^{113} \mathrm{In}$, respectively. The corresponding frequency-gated TOF distributions for ${ }^{113} \mathrm{In}$ (green) and ${ }^{115} \mathrm{In}$ (blue) are shown on the right.

$$
\Delta E_{\mathrm{hf}}=\frac{A_{\mathrm{hf}} C}{2}+\frac{B_{\mathrm{hf}}}{8} \frac{3 C(C+1)-4 I J(I+1)(J+1)}{I J(2 I-1)(2 J-1)},
$$

with $C=F(F+1)-J(J+1)-I(I+1)$, where $F$ takes all values between $|I-J|$ and $I+J$ in integer steps. The isotope shifts $\delta \nu^{A A^{\prime}}$ were extracted from the differences in the hfs centroids, $\delta \nu^{A A^{\prime}}=\nu^{A}-\nu^{A^{\prime}}$, measured for the isotopes $A$ and $A^{\prime}$, respectively.

An example of the fit to a TOF-corrected hfs spectrum measured for the 246.8-nm transition of ${ }^{115} \mathrm{In}$ is shown in Fig. 8. Typically, reduced $\chi^{2}$ values between 1 and 2 were obtained for all of the fitted hfs spectra.

A resolution of about $80 \mathrm{MHz}$ was obtained for zero extraction voltage, and the TOF correction allowed almost perfectly symmetric line shapes to be achieved with FWHMs down to $50 \mathrm{MHz}$ (Fig. 8). The fitted values for

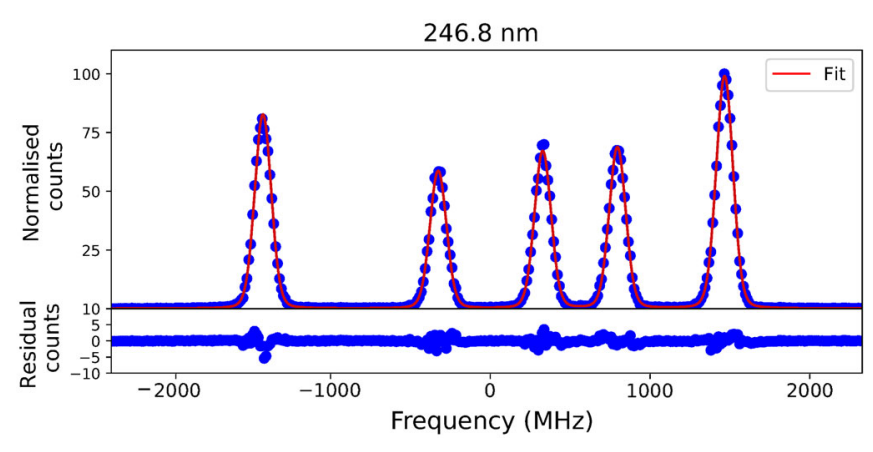

FIG. 8. Fitted hfs spectrum and residuals. Example of a fitted TOF-corrected hfs spectrum measured for ${ }^{115} \mathrm{In}$ in the 246.8-nm transition using "zero" extraction field. The total number of counts are normalized to 100 at the highest peak. The continuous lines show the fit with Voigt profiles. The residuals for the fit are shown on the bottom figure. the hyperfine-structure constants of ${ }^{113} \mathrm{In}$ and ${ }^{115} \mathrm{In}$ are listed in Table I.

To investigate the influence of the TOF correction, the hfs parameters were analyzed for different extraction voltages with and without TOF correction. An example of the results obtained for the 246.8-nm transition of ${ }^{115}$ In is shown in Fig. 9. The extracted hfs parameters for two different extraction fields and respective TOF corrections are compared with our reported values presented in Table I. In general, a good agreement was found among the different approaches, but considerably smaller statistical uncertainties were obtained when the TOF correction was applied.

Our measured hfs values, reported in Table I, are in good agreement with previously reported values for the ${ }^{2} P_{1 / 2,3 / 2}$ states. For the $P_{5 / 2}$, two measurements of the magnetic dipole constant are reported in the literature. Our new results agree with the value reported in Ref. [62], improving the uncertainty of this value by more than 1 order of magnitude. For the ${ }^{113}$ In isotope with a lower natural abundance of $4.3 \%$, less experimental information is available. In the current approach, both isotopes can be clearly separated and measurements can be performed with a low background (see Fig. 6). A complete scan for this isotope was performed with a similar time as for ${ }^{115} \mathrm{In}$ ( $\sim 5 \mathrm{~min}$ ). Signal-to-background ratios of the order of $10^{3}$ and $10^{4}$ were obtained for ${ }^{113} \mathrm{In}$ and ${ }^{115} \mathrm{In}$, respectively.

The mass-separation power can be quantified by the selectivity parameter, $S=(\Delta \omega / \Gamma)^{2}$, which relates the maximum frequency separation between two isotopes $\Delta \omega$ and the resolution of the observed hfs peaks $\Gamma$. We have achieved a selectivity of $2 \times 10^{4}$ at $20 \mathrm{keV}$ for the 246.8-nm transition. As illustrated in the insets of Fig. 3, using traditional RIMS techniques (at rest), the hfs spectra of both isotopes present a large overlap. For the same hfs resolution, the selectivity obtained at $20 \mathrm{keV}$ is $600-2600$ times higher than the value at rest.

A relative isotopic composition of ${ }^{113} \mathrm{In} /{ }^{115} \mathrm{In}=$ $4.30(16) \%$ was obtained from the ratio between the integrated counts in the measured hfs spectra. Although further studies are needed to verify the accuracy that can be reached in the determination of isotopic compositions, our results are very encouraging. This can be compared with standard techniques, e.g., laser ablation-inductively coupled plasma-mass spectrometry (LA-ICP-MS), which reaches a typical precision of a few percent [64]. The determination of isotopic compositions in materials has applications in diverse fields such as environmental sciences, geology, archaeology, biology, and medicine [65-67].

In order to identify other possible sources of systematic uncertainties, the hfs values for each transition were extracted for different experimental conditions as well as different considerations during the data analysis. The latter was affected mainly by different binning choices in time 
TABLE I. Hyperfine-structure values obtained from the fit to the experimental data of ${ }^{113}$ In and ${ }^{115}$ In compared to previous measurements. Statistical (first brackets) and systematic uncertainties (second brackets) are shown independently. Theoretical results for $A_{\mathrm{hf}}$ from the CCSD and CCSD(T) methods are presented. All values are positive except where indicated. The uncertainties $(\Delta)$ of the calculated values are estimated due to the neglected triple excitation configurations given by Eq. (18). Contributions from higher-level excitations in the CCSD method have not been taken into account. These higher-level excitations are expected to be smaller than the triples contributions, but could add sizable contributions to the theoretical uncertainties. The known high-precision values $\mu_{I}=$ $+5.5289(2) \mu_{N}$ [56] and $\mu_{I}=+5.5408(2) \mu_{N}$ [57] of the isotopes ${ }^{113} \mathrm{In}$ and ${ }^{115} \mathrm{In}$, respectively, were used to extract the theoretical $A_{\mathrm{hf}}$ factors from the calculated $A_{\mathrm{hf}} / g_{I}$ values. Both isotopes have nuclear spin $I=9 / 2$.

\begin{tabular}{|c|c|c|c|c|c|c|c|c|}
\hline \multirow[b]{4}{*}{ Level } & \multicolumn{6}{|c|}{$A_{\mathrm{hf}}(\mathrm{MHz})$} & \multirow{2}{*}{\multicolumn{2}{|c|}{$\begin{array}{l}B_{\mathrm{hf}}(\mathrm{MHz}) \\
\text { Experiment }\end{array}$}} \\
\hline & \multicolumn{4}{|c|}{ Theory } & \multicolumn{2}{|c|}{ Experiment } & & \\
\hline & \multicolumn{2}{|c|}{ Literature } & \multicolumn{2}{|c|}{ This work } & \multirow{2}{*}{ Literature [Reference] } & \multirow[t]{2}{*}{ This work } & \multirow[t]{2}{*}{ Literature [Reference] } & \multirow[t]{2}{*}{ This work } \\
\hline & SD [58] & CCSD [20] & CCSD & $\operatorname{CCSD}(\mathrm{T})$ & & & & \\
\hline \multicolumn{9}{|l|}{${ }^{115} \mathrm{In}$} \\
\hline $5 p^{2} P_{1 / 2}$ & 2306 & $2256(30)$ & $2260(30)$ & $2274(25)$ & 2281.9501(4) [59] & $2282.04(45)(53)$ & & \\
\hline $5 p^{2} P_{3 / 2}$ & & & $257(15)$ & 253(10) & $242.1647(3)[59]$ & $241.98(12)(26)$ & $449.545(3)$ [59] & $450(1)(0.5)$ \\
\hline $6 s^{2} S_{1 / 2}$ & 1812 & $1611(50)$ & $1621(50)$ & $1645(37)$ & $1685.3(6)[60]$ & $1684.75(76)(39)$ & & \\
\hline $7 s^{2} S_{1 / 2}$ & 544.5 & $516(30)$ & $510(30)$ & $520(19)$ & $541.0(3)[61]$ & & & \\
\hline $8 s{ }^{2} S_{1 / 2}$ & 240.8 & $234(20)$ & $230(20)$ & $233(10)$ & & $243.85(24)(19)$ & & \\
\hline $9 s^{2} S_{1 / 2}$ & 128.1 & $106(10)$ & 131(10) & $136(6)$ & & 131.07(98)(15) & & \\
\hline $5 s 5 p^{2}{ }^{4} P_{5 / 2}$ & & & & & $\begin{array}{l}3654(13)[61] \\
3689(20)[62]\end{array}$ & $3696(1)(2)$ & $\begin{array}{l}-644(13)[61] \\
-638(50)[62]\end{array}$ & $-660(30)(3)$ \\
\hline \multicolumn{9}{|l|}{${ }^{113}$ In } \\
\hline $5 p^{2} P_{1 / 2}$ & & & $2255(30)$ & $2270(25)$ & $2277.0860(4)$ [59] & $2277.07(34)(77)$ & & \\
\hline $5 p^{2} P_{3 / 2}$ & & & $256(15)$ & $252(10)$ & $241.6409(4)$ [59] & $241.75(25)(21)$ & $443.414(4)[59]$ & $442(3)(1)$ \\
\hline $6 s{ }^{2} S_{1 / 2}$ & & & $1618(50)$ & $1642(37)$ & $1681.8(8)[63]$ & $1682.47(98)(76)$ & & \\
\hline $7 s^{2} S_{1 / 2}$ & & & $509(30)$ & 519(19) & & & & \\
\hline $8 s^{2} S_{1 / 2}$ & & & $229(20)$ & $231(10)$ & & $243.49(24)(23)$ & & \\
\hline $9 s{ }^{2} S_{1 / 2}$ & & & 130(10) & $136(6)$ & & 130.87(94)(38) & & \\
\hline $5 s 5 p^{2}{ }^{4} P_{5 / 2}$ & & & & & & $3690(2)(3)$ & & $-558(35)(5)$ \\
\hline
\end{tabular}

and frequency. Table II shows the parameters that contribute significantly to the extracted isotope shifts and hfs values. The ion energy includes the uncertainty on the acceleration voltage and extraction potential. This parameter presents the largest contribution to the isotope-shift uncertainties. The laser frequency calibration and possible wave meter shifts and nonlinear effects that can be present during the laser scan are listed as "Wave meter cal." This was the main source of systematic errors for the extracted hfs values. The uncertainty due to the possible variations to the binning and fitting procedures was included as "Data analysis" in the same table.

The offset between the time-of-flight distributions of ${ }^{115} \mathrm{In}$ and ${ }^{113} \mathrm{In}, \Delta t$ (see Fig. 7) was used in the line shape correction before extracting the isotope shift. The extracted isotope shifts for different hfs measurements (scans) are shown in Fig. 10 with and without applying the $\Delta t$ correction. As the isotope shifts compare two different masses, a direct measurement of $\Delta t$ is needed. On the other hand, the hfs parameters are extracted for each isotope independently, and do not depend on this offset. The final results for the isotope shifts between ${ }^{115} \mathrm{In}$ and ${ }^{113} \mathrm{In}$ for the different transitions studied are shown in Table III. This work reports the first measurements of the isotope shifts for the 246.0- and 246.8-nm transitions. For the 410.2-nm line, our result is in good agreement with the literature value.

The uncertainty of the reported isotopes shifts is dominated by the uncertainty of the ion-beam energy. Independent high-precision measurements of isotope shifts could be used to calibrate the ion-beam energy and improve considerably the uncertainty of our measurements. Moreover, frequency-comb-based laser spectroscopy with simultaneous collinear and anticollinear experiments could be used to remove the dependence on the beam energy [69]. Our experimental scheme would allow high-precision measurements of isotope shifts over isotope chains of naturally occurring elements. Isotope shifts provide a striking test of many-body methods as they are highly sensitive to electron-correlation effects [70,71]. Recently, isotope shifts measurements were proposed as a method to explore the existence of new forces with unprecedented sensitivity [2,3,72]. High-precision systematic measurements of various atomic transitions in different isotopic chains will be of great relevance in these studies [73]. 


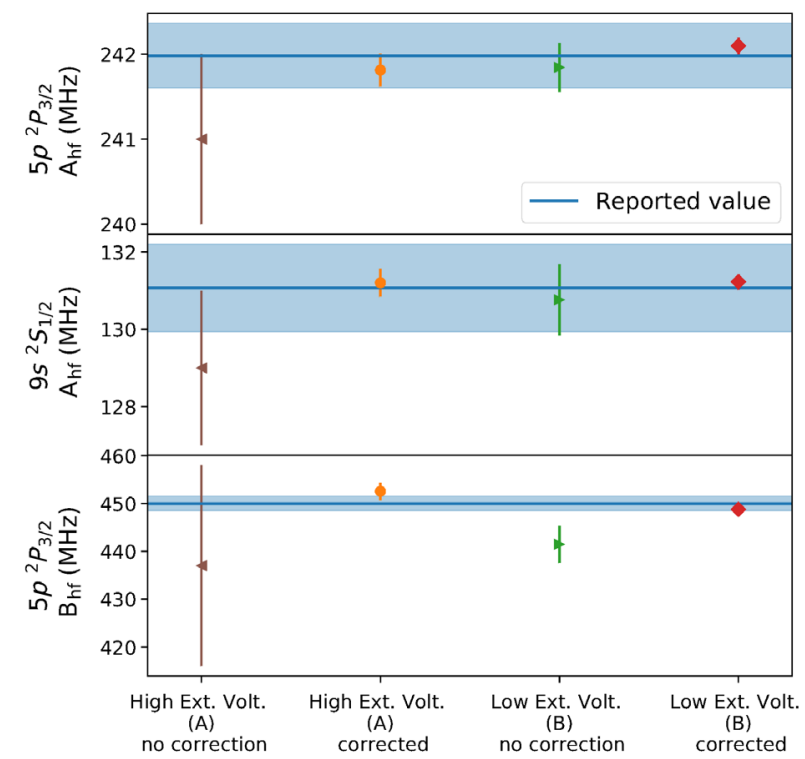

FIG. 9. Example of extracted hfs parameters for different TOF corrections. The hfs parameters of the 246.8-nm transition of ${ }^{115}$ In were analyzed for two different extraction fields, estimated as $5 \mathrm{~V} / \mathrm{mm}$ (high) and $0.02 \mathrm{~V} / \mathrm{mm}$ (low). The results are compared with (corrected) and without (no correction) TOF correction, including statistical uncertainties only. The shadow areas show our final reported values including statistical and systematic uncertainties (see Table I).

\section{Ab initio many-body calculations}

Relativistic coupled-cluster calculations in the singles and doubles excitation approximation (CCSD) were performed, including corrections over the Dirac-Coulomb Hamiltonian due to the Breit and lower-order quantumelectrodynamics interactions (see Sec. III). The calculated values for the ratio between $A_{\mathrm{hf}}$ and the nuclear gyromagnetic factor $g_{I}$, as well as the ratio between $B_{\mathrm{hf}}$ and the nuclear quadrupole moment $Q$ of six low-lying states of In, are listed in Table IV. Results are presented using the DiracHartree-Fock and CCSD methods. Corrections from the
Breit interaction, QED effects, and triples are also quoted explicitly from the CCSD method. As can be seen from the differences between the DHF and CCSD values in Table IV, the degree of electron-correlation effects involved in the determination of the above quantities is different in each state. Especially in the $5 p^{2} P_{3 / 2}$ state, the DHF value for $A_{\mathrm{hf}} / g_{I}$ is larger than the CCSD result, whereas the opposite is true for the other states. The trend is also different in the evaluation of the $B_{\mathrm{hf}} / Q$ value from the $A_{\mathrm{hf}} / g_{I}$ result. Corrections from both the Breit and QED interactions do not change the results substantially. However, we found the triples contributions are significant. The possible uncertainties for the calculated values are presented at the bottom of the table. Contributions from higher-level excitations in the CCSD method have not been taken into account. These higher-level excitations are expected to be smaller than the triples contributions, but could add sizable contributions to the theoretical uncertainties.

\section{Atomic-physics results}

An independent comparison of the calculations with the measured dipole hfs constants is possible by using the wellknown nuclear magnetic moment and spin of each isotope. Theoretical values are compared with the experimental results in Table I. Small nuclear-size effects are neglected in the calculations of the atomic wave functions of ${ }^{113}$ In and ${ }^{115}$ In. Such a comparison cannot be performed for the $B_{\mathrm{hf}}$ constants, as there are no available model-independent measurements of the nuclear quadrupole moment for these isotopes. Overall, we find a good agreement between the experimental results and the calculations including contributions from triples. The uncertainties in the calculations could be further reduced by including full triples excitations.

The errors in the evaluation of weak-interaction Hamiltonians of both PNC and EDM studies are determined by evaluating the off-diagonal matrix elements of the magnetic dipole interaction Hamiltonian, which are

TABLE II. Main sources of systematic errors identified in the extraction of isotope shifts and hfs values.

\begin{tabular}{|c|c|c|c|c|c|c|c|c|c|c|c|}
\hline & \multicolumn{6}{|c|}{$A_{\mathrm{hf}}(\mathrm{MHz})$} & \multicolumn{2}{|c|}{$B_{\mathrm{hf}}(\mathrm{MHz})$} & \multicolumn{3}{|c|}{$\delta \nu^{115,113}(\mathrm{MHz})$} \\
\hline & $5 p^{2} P_{1 /}$ & $5 p^{2} P_{3}$ & $6 s^{2} S_{1 / 2}$ & $8 s^{2} S_{1 / 2}$ & $9 s^{2} S_{1 / 2}$ & $5 p^{2}{ }^{4} P_{5 / 2}$ & $5 p^{2} P_{3 / 2}$ & $s 5 p^{2}{ }^{4} P_{5 / 2}$ & $246.0 \mathrm{~nm}$ & $246.8 \mathrm{~nm}$ & $410.2 \mathrm{~nm}$ \\
\hline \multicolumn{12}{|l|}{${ }^{113} \mathrm{In}$} \\
\hline Ion energy & 0.10 & 0.04 & 0.16 & 0.06 & 0.04 & 0.82 & 0.09 & 2 & & & \\
\hline Wave meter cal. & 0.74 & 0.19 & 0.07 & 0.15 & 0.24 & 3.1 & 0.82 & 4 & & & \\
\hline Data analysis & 0.19 & 0.09 & 0.74 & 0.17 & 0.29 & 1.2 & 1.10 & 3 & & & \\
\hline $\begin{array}{l}\text { Total error } \\
{ }^{115} \mathrm{In}\end{array}$ & 0.77 & 0.21 & 0.76 & 0.23 & 0.38 & 3.42 & 1.37 & 5 & & & \\
\hline Ion energy & 0.02 & 0.004 & 0.12 & 0.006 & 0.016 & 0.3 & 0.01 & 0.20 & 3.7 & 4.1 & 4.8 \\
\hline Wave meter cal. & 0.53 & 0.26 & 0.37 & 0.19 & 0.14 & 2.3 & 0.44 & 2.6 & 2.1 & 4 & 1.5 \\
\hline Data analysis & 0.04 & 0.016 & 0.031 & 0.021 & 0.044 & 0.4 & 0.15 & 1.8 & 0.46 & 0.13 & 0.53 \\
\hline$\Delta t$ & & & & & & & & & 1.6 & 2.7 & 1.3 \\
\hline Total error & 0.53 & 0.26 & 0.39 & 0.19 & 0.15 & 2.3 & 0.46 & 3.2 & 4.6 & 6.3 & 5.2 \\
\hline
\end{tabular}






FIG. 10. Measured isotope shifts. Isotope shifts between ${ }^{115} \mathrm{In}$ and ${ }^{113}$ In obtained for the 246.0-nm (a), 246.8-nm (b), and 410.0-nm (c) transitions from different measurements (scans). The measured offset $(\Delta t)$ between the time-of-flight distributions of ${ }^{115}$ In and ${ }^{113}$ In (see Fig. 7) was used to extract the isotope shifts for each transition. Results are shown with (orange) and without (blue) applying the $\Delta t$ correction. The average values are shown with statistical uncertainties only.

assumed to be proportional to square-root values of the $A_{\mathrm{hf}}$ values of the associated states in the matrix elements $[20,21,29,74]$. In the indium atom, the $A_{\mathrm{hf}}$ values of the $5{ }^{2} P_{1 / 2}$ and $6-9 s{ }^{2} S_{1 / 2}$ states were shown to provide the leading contributions to assess the accuracy of these

TABLE III. Measured isotope shifts for the 246.0-, 246.8-, and 410.2-nm transitions. Statistical (first brackets) and systematic uncertainties (second brackets) are shown independently.

\begin{tabular}{lccc}
\hline \hline & & $\delta \nu^{115,113}(\mathrm{MHz})$ & \\
$\lambda(\mathrm{nm})$ & Transition & Literature [Ref.] & This work \\
\hline 246.0 & $5 p{ }^{2} P_{1 / 2} \rightarrow 8 s{ }^{2} S_{1 / 2}$ & & $268(2)(5)$ \\
246.8 & $5 p{ }^{2} P_{3 / 2} \rightarrow 9 s{ }^{2} S_{1 / 2}$ & & $-270(2)(6)$ \\
410.2 & $5 p{ }^{2} P_{1 / 2} \rightarrow 6 s{ }^{2} S_{1 / 2}$ & $-258(3)[68]$ & $-260(3)(5)$ \\
\hline \hline
\end{tabular}

TABLE IV. Calculated values of $A_{\mathrm{hf}} / g_{I}$ and $B_{\mathrm{hf}} / Q$ in $\mathrm{MHz}$ for low-lying states of In. Results from the DHF and CCSD methods are given separately. Corrections due to the Breit, QED effects, and triples over the DC Hamiltonian from the CCSD method are quoted explicitly. Estimated uncertainties to each quantity are also given. Contributions from higher-level excitations in the CCSD method have not been taken into account (see text for more details).

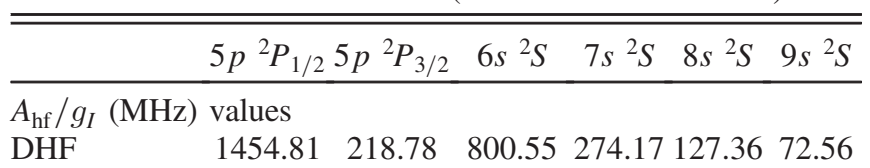

Values from CCSD method

\begin{tabular}{lrrrrrr} 
DC & 1841.52 & 207.96 & 1321.11 & 415.95 & 187.41 & 106.88 \\
Breit & -2.67 & 0.10 & 1.61 & 0.64 & 0.30 & 0.15 \\
QED & -3.11 & 0.78 & -5.81 & -2.11 & -1.08 & -0.78 \\
Triples & 11.27 & -3.68 & 19.07 & 7.87 & 2.59 & 1.46 \\
Uncertainty \pm & 20.0 & 8.0 & 30.0 & 15.0 & 8.0 & 3.0 \\
$B_{\text {hf }} / Q(\mathrm{MHz} / \mathrm{b})$ & values & & & & & \\
DHF & 419.83 & & & & \\
Values from CCSD method & & & & & \\
DC & 581.61 & & & & \\
Breit & -2.27 & & & \\
QED & 0.04 & & & \\
Triples & -3.78 & & & \\
Uncertainty \pm & 4.0 & & & \\
\hline
\end{tabular}

Hamiltonians [20]. Our new results for $8 s{ }^{2} S_{1 / 2}$ and $9 s \quad{ }^{2} S_{1 / 2}$ complete the experimental knowledge for all relevant states in this atom. Theoretical predictions for these states in ${ }^{115}$ In were reported in Ref. [20] using the CCSD method with the DC Hamiltonian and considering Gaussian-type orbitals (GTOs). The present theoretical calculations differ slightly in all of the states; however, a significant difference is observed for the $9 s^{2} S_{1 / 2}$ state. Our theoretical results reported here are in good agreement with the new measurements. The small differences between the calculations are mainly due to two reasons: the inclusion of higher relativistic corrections and the inclusion of higher nonlinear terms [from $\bar{O}$ and $\overline{\mathcal{N}}$ in Eq. (17)] through iterative procedures that were truncated only at the linear terms in $T$ and $T^{\dagger}$ in Ref. [20] (see Sec. III). The reason for the large discrepancy in the $9 s^{2} S_{1 / 2}$ is mostly due to the use of quadratic-type orbitals (QTOs) instead of GTOs as basis functions. Since this state is close to the continuum, it has a larger overlap with the nucleus and the QTOs are able to describe its wave function better than the GTOs.

Additional theoretical calculations have reported $A_{\mathrm{hf}}$ values for the ground and excited states in ${ }^{115}$ In only considering the linear terms from the CCSD method (SD method) [58]. We find significant differences between the results from the SD and CCSD methods (see Table I). This implies that including nonlinear terms of the RCC method is very important to accurately describe electron-correlation effects in the indium atom. 
TABLE V. Nuclear magnetic dipole and electric quadrupole moments derived from the measured hyperfine-structure constants. The calculated $A_{\mathrm{hf}} / g_{I}=+1847(20) \mathrm{MHz}$ factor was used to extract the magnetic moments from the measured $A_{\mathrm{hf}}\left({ }^{2} P_{1 / 2}\right)$ value.

\begin{tabular}{lccccc}
\hline \hline & \multicolumn{3}{c}{${ }^{113} \mathrm{In}$} & & \multicolumn{2}{c}{${ }^{115} \mathrm{In}$} \\
\cline { 2 - 3 } \cline { 5 - 6 } & Literature & This & & Literature & This \\
[Ref.] & work & & [Ref.] & work \\
\hline$\mu_{I}\left(\mu_{N}\right)$ & $5.5289(2)[56]$ & $5.548(62)$ & $5.5408(2)[57]$ & $5.560(62)$ \\
$Q(\mathrm{~b})$ & $0.80(4)[60]$ & $0.767(11)^{\mathrm{a}}$ & $( \pm) 0.83(10)[76]$ & $0.781(7)^{\mathrm{a}}$ \\
& & $0.770(5)^{\mathrm{b}}$ & $( \pm) 0.770(8)[77]$ & $0.780(5)^{\mathrm{b}}$ \\
\hline \hline
\end{tabular}

${ }^{\mathrm{a} Q u a d r u p o l e ~ m o m e n t s ~ w e r e ~ e x t r a c t e d ~ u s i n g ~ o u r ~ c a l c u l a t e d ~}$ $B_{\mathrm{hf}} / Q=+576(4) \mathrm{MHz} / \mathrm{b}$ factor with the experimental $B_{\mathrm{hf}}\left({ }^{2} P_{3 / 2}\right)$ value obtained from our measurement.

${ }^{\mathrm{b}}$ From previous high-precision values from Refs. [59,78]. All values are positive except where indicated.

\section{E. Nuclear structure results}

Nuclear magnetic dipole moments and electric quadrupole moments can be extracted from the measured hyperfine-structure constants, provided that the atomic-physics part is known. The calculated $A_{\mathrm{hf}} / g_{I}=1847(20) \mathrm{MHz}$ value (Table IV) was used to obtain the magnetic moments from the measured $A_{\mathrm{hf}}\left({ }^{2} P_{1 / 2}\right)$ parameters, neglecting the small hyperfine anomaly in the indium atom, which is less than $0.003 \%$ [75]. The results for the nuclear moments are listed in Table V. The extracted nuclear magnetic moments for ${ }^{113} \mathrm{In}$ and ${ }^{115} \mathrm{In}$ are in good agreement with the literature values.

The calculated $B_{\mathrm{hf}} / Q$ factor was used to extract the nuclear quadrupole moments from the measured $B_{\mathrm{hf}}\left({ }^{2} P_{3 / 2}\right)$ factors for both isotopes. The calculated result for this factor is presented in Table IV. The final value after Breit and QED corrections is reported as $B_{\mathrm{hf}} / Q=$ $576(4) \mathrm{MHz} / \mathrm{b}$, including the uncertainties from the contributions of neglected triple excitations in the coupled-cluster expansion. The extracted nuclear quadrupole moments are included in Table V. For ${ }^{115} \mathrm{In}$, the previous most precise value for the quadrupole moment was determined using quantum-chemical calculations from a measurement of the quadrupole-coupling constant in a molecule [77]. As seen from Table V, our results agree with the literature values, and the uncertainty of the quadrupole moments for both isotopes has been improved by combining our new theoretical calculations and high-precision measurements. The uncertainties on the extracted nuclear observables is mainly dominated by the uncertainties of the theoretical calculations.

The 246.8-nm transition studied in this work was shown to be sensitive to both nuclear magnetic moments and electric quadrupole moments. Moreover, it exhibits considerable advantages with respect to the other transitions previously studied. The hfs parameters $A_{\mathrm{hf}}$ and $B_{\mathrm{hf}}$ have a similar magnitude and are large enough to be measured with relative uncertainties lower than $1 \%$ and $5 \%$, respectively. Furthermore, the relatively small hyperfine structure $(<5 \mathrm{GHz})$ of this transition is ideal for experiments with radioactive isotopes, which are typically performed over a period of only a few days. Scanning speeds as low as $10 \mathrm{MHz} / \mathrm{min}$ are needed for the study of exotic radioactive isotopes, which can be produced with yields of just a few ions/s. While a complete scan of the 246.8-nm transition would take about 8.3 hours at this scanning speed, more than 24 hours would be needed for transitions with a splitting larger than $15 \mathrm{GHz}$, e.g., 283.7-nm transition. This considerable reduction of scanning time is decisive for online experiments with radioactive isotopes that must cope with the short-time availability at radioactive-beam facilities. An experimental research program that will make use of this transition for the study of exotic indium isotopes is ongoing at ISOLDE-CERN [32].

\section{FUTURE PERSPECTIVES}

The experimental method as presented here can be applied to study atomic-physics parameters of other elements of fundamental-physics interest such as $\mathrm{Cs}$, $\mathrm{Tl}$, and $\mathrm{Hg}$. By varying the time delay between the probe laser and the ionization pulse, and taking the normalized measurements for each ion bunch, lifetime measurements of atomic states could also be performed. Such lifetime measurements can provide valuable complementary information for atomic-physics theory and testing of weak-interaction Hamiltonians [79]. These studies can be extended to ions in different charge states created in the laser-ablation process. A unique advantage of the present approach is the possibility of studying high-lying atomic states, which can be accessed using metastable states populated in flight after the neutralization process. It opens up the possibility to perform experiments on elements that have not been studied yet by high-precision laser spectroscopy, e.g., carbon isotopes. These isotopes are of the utmost importance for nuclear structure [80-82] as well as Earth and environmental sciences [83], but are out of reach for the current laser-spectroscopy methods. For these isotopes the transitions from the ground states lie in the extreme ultraviolet region, which is not accessible by available high-resolution laser technology. However, after neutralization with a vapor gas, a small fraction of the ion beam would be neutralized into a high-lying metastable atomic state, from which atomic transitions in laser accessible ranges are available. This neutralization process and subsequent population of different atomic states provides a means of preparing atomic states of interests for hfs measurements.

The possibility of performing the spectroscopy directly on pulses of ablated ions without the use of an ion trap presents a major advantage in the study of light elements. Light elements are particularly challenging for ion trapping for several reasons. In addition to be highly reactive, the 
stability parameter of radio-frequency traps is inversely proportional to the ion mass [84]. Therefore, larger radio frequencies are needed, which can increase the kinetic energy of the ions and reduce the trapping efficiency [85]. Additionally, the requirement of $\mathrm{H}_{2}$ as a buffer gas to efficiently trap light elements imposes major technical difficulties.

Our experimental method can be applied to investigate the structure of molecular species. It has been shown that molecular systems provide suitable physical systems for studies of fundamental symmetries with unprecedented sensitivity [9,86-89]. However, the experimental knowledge of molecules is scarce, and in most of the cases, quantum-chemistry calculations constitute the only source of information. Fluoride molecules, for example, can be produced by laser ablation. This, combined with the high efficiency of the collinear resonance ionization method and the use of broadband and narrow-band pulsed lasers, would allow one to scan large frequency spaces in realistic short timescales, typically of a few days only. Such measurements can provide excitation energies, ionization potentials, and molecular hyperfine-structure parameters to benchmark quantum-chemistry calculations and support the development of laser-cooling schemes for higher precision studies [90]. Although advances have been made in the spectroscopy of molecules, very little is known about the fine and hyperfine molecular coupling constants as well as their isotopic dependence [91]. Our approach would offer unique advantages in these studies, as it can be applied to both neutral and ionic molecules with different isotopic compositions. The high sensitivity of our method is crucial to study complex molecular structures that are not accessible by optical techniques due to the broad distribution of states. Currently, several neutral molecules are of great interest for fundamental physics studies whose lasercooling schemes remain unknown [92]. Moreover, our approach could provide access to study ionic molecular states that are not accessible within ion traps, e.g., due to unavoidable distributions of states within the trap [93].

As shown in this work, the hfs spectra of the two stable indium isotopes were clearly separated and measured with low-background conditions. Moreover, at $20 \mathrm{keV}$, a gain in selectivity of more than 2 orders of magnitude is obtained with respect to traditional RIMS techniques. These results present a step forward to reach the predicted sensitivity of the collinear resonance ionization approach, to perform $\mathrm{hfs}$ measurements in elements with relative abundances as low as $1: 10^{10}$ [94]. A particle detector in the atom path will allow normalization of the ion-beam current for individual ion bunches. This can be used as a normalization factor to correct any ion-source instability. Such a normalization can be very important for measurements that require knowledge of the ion count rate at different times, e.g., lifetime measurements of atomic states and relative isotope abundance. This type of neutral detector, which is capable of operating in the presence of high-power laser pulses, has been developed for the study of negative ions [95]. A precise determination of the concentration of lowabundance elements in low-background conditions has a direct impact in geophysics and cosmochronology studies [96-99].

The understanding of laser-generated plasmas has gained attention due to its importance for studies of laser-matter interaction in strong fields [100-104] as well as its applications to different research areas [105-107]. By performing high-resolution hfs measurements on wellknown transitions of atomic species generated in the ablation process, properties of the laser-generated plasma can be extracted. As the profile and width of the measured hfs peaks are sensitive to the initial conditions of the ablated ions, this approach can be used to determine their velocity distribution. Simultaneous measurements of the TOF and the ion-beam energy spread could offer complementary information to investigate the ablation process. These experiments would also allow a precise identification of the ablated products that are created in different charge states. Systematic studies of these parameters and their influence in the observed hfs spectra for different laser fluences, laser wavelengths, and pulse duration on different ablation materials will be investigated in a forthcoming publication.

\section{CONCLUSIONS}

We demonstrate that the use of accelerated laser-ablated ions avoids the technical complexity of ion traps and massseparation devices, considerably simplifying the application of the CRIS approach, and placing the experimental method at the scale of tabletop experiments. This provides major and unique advantages with respect to existing methods: (i) the experimental method can be applied to both atoms and molecules in both neutral and ionic states, (ii) it provides significant advantages to study elements that are highly reactive, for which the production and trapping are particularly challenging, (iii) in-flight neutralization allows high-lying metastable states to be studied, which are not easily accessible by other means, (iv) spectroscopy can be performed directly on highly intense ion bunches $\left(>10^{6}\right.$ ions/bunch) created by laser-generated plasmas, which would be unfeasible using ion traps due to space-charge limitations, (v) these properties combined with the high selectivity, low background, and high efficiency open up new opportunities to perform high-precision experiments on a wide range of elements and on isotopes with extremely low natural abundances. This is critical for applications in molecular spectroscopy and trace analysis studies.

Despite the energy spread of the probed ion beam, highresolution measurements were achieved by applying a TOF correction to the observed hyperfine-structure line shapes. To our knowledge, this is the first time that this approach has been implemented with collinear laser spectroscopy. 
These results open up the possibility of performing highprecision studies under different laser-ablation conditions, and could provide further insights in the study of lasergenerated plasmas. The method of TOF-corrected line shapes can be extended to perform high-precision studies on elements produced by other mechanisms with large energy spread, e.g., atoms or ions created in flight from the breakup of a molecular beam. Additionally, this method could offer a new alternative when performing highprecision studies on radioactive elements that are created in flight or extracted mainly as molecular species.

The importance of our results in nuclear, atomic, and fundamental physics was demonstrated by performing, for the first time, hyperfine-structure measurements of selected states of the indium atom. The magnetic dipole hyperfinestructure constants of the $8 s^{2} S_{1 / 2}$ and $9 s^{2} S_{1 / 2}$ states of the two naturally occurring indium isotopes, ${ }^{113,115} \mathrm{In}$, as well as the magnetic dipole and electric quadrupole hyperfinestructure constants of the $5 s 5 p^{2}{ }^{4} P_{5 / 2}$ state of ${ }^{113} \mathrm{In}$, are measured for the first time. Additionally, we present the first measurements of the isotope shifts between ${ }^{113} \mathrm{In}$ and ${ }^{115}$ In in the 246.0- and 246.8-nm transitions. The importance of the new experimental results in the development of atomic many-body methods is highlighted by comparing with theoretical predictions. Relativistic coupled-cluster calculations in the singles and doubles excitation approximation are performed, including corrections over the Dirac-Coulomb Hamiltonian due to the Breit, triples, and lower-order quantum electrodynamic interactions. Our new measurement for the dipole hyperfine-structure constants of the $9 s^{2} S_{1 / 2}$ state is shown to be particularly sensitive to the details of the atomic-physics calculations. These findings are important to test the reliability of manybody methods that are employed to determine matrix elements of the weak interactions in PNC and EDM studies. Benchmarked atomic-physics calculations are used to provide an accurate value of $B_{\mathrm{hf}} / Q$ for the ${ }^{2} P_{3 / 2}$ state of the indium atom, allowing the extraction of accurate nuclear quadrupole moments of ${ }^{113} \mathrm{In}$ and ${ }^{115} \mathrm{In}$ from measurements of electric quadrupole hfs constants. These results establish new and reliable reference values for the extraction of nuclear quadrupole moments from hyperfine-structure measurements of radioactive indium isotopes. From all of the atomic transitions that have been studied in the indium atom, the 246.8-nm line studied in this work is shown to provide marked advantages that will be decisive for future studies of short-lived indium isotopes.

\section{ACKNOWLEDGMENTS}

This work was supported by ERC Consolidator Grant No. 648381 (FNPMLS); STFC Grants No. ST/L005794/1 and No. ST/L005786/1; GOA 15/010 from KU Leuven; the FWO-Vlaanderen (Belgium) and the European Union's
Horizon 2020 research and innovation programme under Grant Agreement No. 654002 (ENSAR 2). We acknowledge the financial aid from the Ed Schneiderman Fund at New York University. B.K.S. acknowledges financial support from Chinese Academy of Science through the PIFI fellowship under Project No. 2017VMB0023 and partly by the TDP project of Physical Research Laboratory (PRL), Ahmedabad, and the computations were carried out using the Vikram-100 HPC cluster of PRL. We would like to thank the ISOLDE technical group for their support and assistance, and the University of Jyväskylä for the use of the injection-locked cavity and data acquisition card.

[1] M. S. Safronova, D. Budker, D. DeMille, D. F. Jackson Kimball, A. Derevianko, and C. W. Clark, Search for New Physics with Atoms and Molecules, Rev. Mod. Phys. 90, 025008 (2018).

[2] J. C. Berengut et al., Probing New Long-Range Interactions by Isotope Shift Spectroscopy, Phys. Rev. Lett. 120, 091801 (2018).

[3] Y. V. Stadnik, V. A. Dzuba, and V. V. Flambaum, Improved Limits on Axionlike-Particle-Mediated P, T-Violating Interactions between Electrons and Nucleons from Electric Dipole Moments of Atoms and Molecules, Phys. Rev. Lett. 120, 013202 (2018).

[4] R. F. Garcia Ruiz et al., Ground-State Electromagnetic Moments of Calcium Isotopes, Phys. Rev. C 91, 041304(R) (2015).

[5] R. F. Garcia Ruiz et al., Unexpectedly Large Charge Radii of Neutron-Rich Calcium Isotopes, Nat. Phys. 12, 594 (2016).

[6] J. Enjerl, M. J. Ramsey-Musolf, and U. van Kolck, Electric Dipole Moments of Nucleons, Nuclei, and Atoms: The Standard Model and Beyond, Prog. Part. Nucl. Phys. 71, 21 (2013).

[7] M. L. Bissell et al., Cu Charge Radii Reveal a Weak SubShell Effect at $N=40$, Phys. Rev. C 93, 064318 (2016).

[8] B. K. Sahoo and B. P. Das, Relativistic Normal CoupledCluster Theory for Accurate Determination of Electric Dipole Moments of Atoms: First Application to the ${ }^{199} \mathrm{Hg}$ Atom, Phys. Rev. Lett. 120, 203001 (2018).

[9] W. B. Cairncross, D. N. Gresh, M. Grau, K. C. Cossel, T. S. Roussy, Y. Ni, Y. Zhou, J. Ye, and E. A. Cornell Precision Measurement of the Electron's Electric Dipole Moment Using Trapped Molecular Ions, Phys. Rev. Lett. 119, 153001 (2017).

[10] J. Lim, J. R. Almond, M. A. Trigatzis, J. A. Devlin, N. J. Fitch, B. E. Sauer, M. R. Tarbutt, and E. A. Hinds, Laser Cooled YbF Molecules for Measuring the Electron's Electric Dipole Moment, Phys. Rev. Lett. 120, 123201 (2018).

[11] K. T. Flanagan et al., Collinear Resonance Ionization Spectroscopy of Neutron-Deficient Francium Isotopes, Phys. Rev. Lett. 111, 212501 (2013).

[12] R. F. Garcia Ruiz et al., Development of a Sensitive Setup for Laser Spectroscopy Studies of Very Exotic Calcium Isotopes, J. Phys. G 44, 044003 (2017). 
[13] R. Ferrer et al., Towards High-Resolution Laser Ionization Spectroscopy of the Heaviest Elements in Supersonic Gas Jet Expansion, Nat. Commun. 8, 14520 (2017).

[14] B. N. Chichkov, C. Momma, S. Nolte, F. Alvensleben, and A. Tünnermann, Femtosecond, Picosecond and Nanosecond Laser Ablation of Solids, Appl. Phys. A 63, 109 (1996).

[15] P. R. Willmott and J. R. Huber, Pulsed Laser Vaporization and Deposition, Rev. Mod. Phys. 72, 315 (2000).

[16] P. Balling and J. Schou, Femtosecond-Laser Ablation Dynamics of Dielectrics: Basics and Applications for Thin Films, Rep. Prog. Phys. 76, 036502 (2013).

[17] R. P. de Groote et al., Use of a Continuous Wave Laser and Pockels Cell for Sensitive High-Resolution Collinear Resonance Ionization Spectroscopy, Phys. Rev. Lett. 115, 132501 (2015).

[18] C. Schulz et al., Resonance Ionization Spectroscopy on a Fast Atomic Ytterbium Beam, J. Phys. B 24, 4831 (1991).

[19] K. M. Lynch et al., Decay-Assisted Laser Spectroscopy of Neutron-Deficient Francium, Phys. Rev. X 4, 011055 (2014).

[20] B. K. Sahoo, R. Pandey, and B. P. Das, Search for a Permanent Electric-Dipole Moment Using Atomic Indium, Phys. Rev. A 84, 030502(R) (2011).

[21] H. S. Nataraj, B. K. Sahoo, B. P. Das, and D. Mukherjee, Intrinsic Electric Dipole Moments of Paramagnetic Atoms: Rubidium and Cesium, Phys. Rev. Lett. 101, 033002 (2008).

[22] H. S. Nataraj, B. K. Sahoo, B. P. Das, and D. Mukherjee, Reappraisal of the Electric Dipole Moment Enhancement Factor for Thallium, Phys. Rev. Lett. 106, 200403 (2011).

[23] S. G. Porsev, M. S. Safronova, and M. G. Kozlov, Electric Dipole Moment Enhancement Factor of Thallium, Phys. Rev. Lett. 108, 173001 (2012).

[24] B. Augenbraun, A. Carter, P. M. Rupasinghe, and P. K. Majumder, Measurement of the Scalar Polarizability of the Indium $6 p_{1 / 2}$ State Using Two-Step Atomic-Beam Spectroscopy, Phys. Rev. A 94, 022515 (2016).

[25] N. B. Vilas, B.-Y. Wang, P. M. Rupasinghe, D. L. Maser, M. S. Safronova, U. I. Safronova, and P. K. Majumder, High-Precision Measurements and Theoretical Calculations of Indium Excited-State Polarizabilities, Phys. Rev. A 97, 022507 (2018).

[26] P. A. Vetter, D. M. Meekhof, P. K. Majumder, S. K. Lamoreaux, and E. N. Fortson, Precise Test of Electroweak Theory from a New Measurement of Parity Nonconservation in Atomic Thallium, Phys. Rev. Lett. 74, 2658 (1995).

[27] N. H. Edwards, S. J. Phipp, P.E. G. Baird, and S. Nakayama, Precise Measurement of Parity Nonconserving Optical Rotation in Atomic Thallium, Phys. Rev. Lett. 74, 2654 (1995).

[28] B. C. Regan, E. D. Commins, C. J. Schmidt, and D. DeMille, New Limit on the Electron Electric Dipole Moment, Phys. Rev. Lett. 88, 071805 (2002).

[29] J.S. M. Ginges and V. V. Flambaum, Violations of Fundamental Symmetries in Atoms and Tests of Unification Theories of Elementary Particles, Phys. Rep. 397, 63 (2004).
[30] C. B. Hinke et al., Superallowed Gamow-Teller Decay of the Doubly Magic Nucleus ${ }^{100} \mathrm{Sn}$, Nature (London)486, 341 (2012).

[31] J. Taprogge et al., $1 p_{3 / 2}$ Proton-Hole State in ${ }^{132} \mathrm{Sn}$ and the Shell Structure Along $N=82$, Phys. Rev. Lett. 112, 132501 (2014).

[32] R. F. Garcia Ruiz et al., Laser Spectroscopy of Exotic Indium $(Z=49)$ Isotopes: Approaching the $N=50$ and $N=82$ Neutron Numbers, Report No. CERNINTC-2017-025, 2017.

[33] R. Kirchner, On the Thermoionization in Hot Cavities, Nucl. Instrum. Methods Phys. Res, Sect. A 292, 203 (1990).

[34] S. Olmschenk and P. Becker, Laser Ablation Production of Ba, Ca, Dy, Er, La, Lu, and Yb Ions, Appl. Phys. B 123, 99 (2017).

[35] L. Torrisi, S. Gammino, L. Andò, V. Nassisi, D. Doria, and A. Pedone, Comparison of Nanosecond Laser Ablation at 1064 and $308 \mathrm{~nm}$ Wavelength, Appl. Surf. Sci. 210, 262 (2003).

[36] B. Toftmann, J. Schou, and S. Canulescu, Energy Distribution of Ions Produced by Laser Ablation of Silver in Vacuum, Appl. Surf. Sci. 278, 273 (2013).

[37] K. K. Anoop, M. P. Polek, R. Bruzzese, S. Amoruso, and S. S. Harilal, Multidiagnostic Analysis of Ion Dynamics in Ultrafast Laser Ablation of Metals Over a Large Fluence Range, J. Appl. Phys. 117, 083108 (2015).

[38] V. Sonnenschein, Ph.D. thesis, The University of Jyväskylä, 2015.

[39] V. Sonnenschein, I. D. Moore, S. Raeder, M. Reponen, H. Tomita, and K. Wendt, Characterization of a Pulsed Injection-Locked Ti:Sapphire Laser and Its Application to High Resolution Resonance Ionization Spectroscopy of Copper, Laser Phys. 27, 085701 (2017).

[40] P. Campbell, I. D. Moore, and M. R. Pearson, Laser Spectroscopy for Nuclear Structure Physics, Prog. Part. Nucl. Phys. 86, 127 (2016).

[41] A. Voss, T. J. Procter, O. Shelbaya, P. Amaudruz, F. Buchinger, J. E. Crawford, S. Daviel, E. Mané, M. R. Pearson, and W. Al Tamimi, The Collinear Fast Beam Laser Spectroscopy (CFBS) Experiment at TRIUMF, Nucl. Instrum. Methods Phys. Res., Sect. A 811, 57 (2016).

[42] J. Levine, M. R. Savina, T. Stephan, N. Dauphas, A. M. Davis, K. B. Knight, and M. J. Pellin, Resonance Ionization Mass Spectrometry for Precise Measurements of Isotope Ratios, Int. J. Mass Spectrom. 288, 36 (2009).

[43] C. Schwartz, Theory of Hyperfine Structure, Phys. Rev. 97, 380 (1955).

[44] B. K. Sahoo, Conforming the Measured Lifetimes of the $5 d^{2} D_{3 / 2,5 / 2}$ States in Cs with Theory, Phys. Rev. A 93, 022503 (2016).

[45] B. K. Sahoo, D. K. Nandy, B. P. Das, and Y. Sakemi, Correlation Trends in the Hyperfine Structures of ${ }^{210,212} \mathrm{Fr}$, Phys. Rev. A 91, 042507 (2015).

[46] D. K. Nandy and B. K. Sahoo, Development of a Relativistic Coupled-Cluster Method for One-Electron Detachment Theory: Application to Mn IX, Fe X, Co XI, and Ni XII Ions, Phys. Rev. A 88, 052512 (2013). 
[47] D. K. Nandy and B. K. Sahoo, Quadrupole Shifts for the ${ }^{171} \mathrm{Yb}^{+}$Ion Clocks: Experiments versus Theories, Phys. Rev. A 90, 050503 (2014).

[48] P. Kumar, C. Li, and B. K. Sahoo, Diverse Trends of Electron Correlation Effects for Properties with Different Radial and Angular Factors in an Atomic System: A Case Study in $\mathrm{Ca}^{+}$, J. Phys. B 51, 055101 (2018).

[49] C.-B. Li, Y.-M. Yu, and B. K. Sahoo, Relativistic CoupledCluster-Theory Analysis of Energies, Hyperfine-Structure Constants, and Dipole Polarizabilities of $\mathrm{Cd}^{+}$, Phys. Rev. A 97, 022512 (2018).

[50] COMSOL MULTIPHYSICS, https://www.comsol.com/comsolmultiphysics.

[51] L. Zhigilei, P. B. S. Kodali, and B. J. Garrison, A Microscopic View of Laser Ablation, J. Phys. Chem. B 102, 2845 (1998).

[52] J. Maul, S. Karpuk, and G. Huber, Bimodal Velocity Distribution of Atoms Released from Nanosecond Ultraviolet Laser Ablation, Phys. Rev. B 71, 045428 (2005).

[53] X. Wang, S. Zhang, X. Cheng, E. Zhu, W. Hang, and B. Huang, Ion Kinetic Energy Distributions in LaserInduced Plasma, Spectrochim. Acta B Atom. Spectros. 99, 101 (2014).

[54] M. Hori and A. Dax, Chirp-Corrected, Nanosecond Ti: Sapphire Laser with $6 \mathrm{MHz}$ Linewidth for Spectroscopy of Antiprotonic Helium, Opt. Lett. 34, 1273 (2009).

[55] W. Gins, R. P. de Groote, M. L. Bissell, C. Granados Buitrago, R. Ferrer, K. M. Lynch, G. Neyens, and S. Sels, Analysis of Counting Data: Development of the Satlas PYTHON Package, Comput. Phys. Commun. 222, 286 (2018).

[56] M. Rice and R. V. Pound, Ratio of the Magnetic Moments of ${ }^{115}$ In and ${ }^{113}$ In, Phys. Rev. 106, 953 (1957).

[57] C. P. Flynn and E. F. W. Seymour, Knight Shift of the Nuclear Magnetic Resonance in Liquid Indium, Proc. Phys. Soc. London 76, 301 (1960).

[58] U. I. Safronova, M. S. Safranova, and M. G. Kozlov, Relativistic All-Order Calculations of In I and Sn II Atomic Properties, Phys. Rev. A 76, 022501 (2007).

[59] T. G. Eck and P. Kusch, Hfs of the $5^{2} P_{\frac{3}{2}}$ State of ${ }^{115}$ In and ${ }^{113}$ In: Octupole Interactions in the Stable Isotopes of Indium, Phys. Rev. 106, 958 (1957).

[60] J. Eberz et al., Spins, Moments and Mean Square Charge Radii of ${ }^{104-127}$ In Determined by Laser Spectroscopy, Nucl. Phys. A464, 9 (1987).

[61] S. George, J. Verges, and G. Guppy, Newly Observed Lines and Hyperfine Structure in the Infrared Spectrum of Indium Obtained by Using a Fourier-Transform Spectrometer, J. Opt. Soc. Am. B 7, 249 (1990).

[62] Y. Y. Zhao, J. Carlsson, H. Lundberg, and C. G. Wahlström, Hyperfine Structure Studies in ${ }^{2} \mathrm{D}$ and ${ }^{4} \mathrm{P}$ States of ${ }^{27} \mathrm{Al},{ }^{69,71} \mathrm{Ga}$ and ${ }^{115} \mathrm{In}, \mathrm{Z}$. Phys. D 3, 365 (1986).

[63] D. A. Jackson, Hyperfine Structure and Isotope Shifts in the Lines $4033 \AA$ of Gallium I and $4101 \AA$ of Indium I, Physica 103 B+C, 437 (1981).

[64] S. E. Jackson, N. J. Pearson, W. L. Griffin, and E. A. Belousova, The Application of Laser Ablation-Inductively Coupled Plasma-Mass Spectrometry to In Situ U-Pb Zircon Geochronology, Chem. Geol. 211, 47 (2004).
[65] Y. Liu, Z. Hu, S. Gao, D. Günther, J. Xu, C. Gao, and H. Chen, In Situ Analysis of Major and Trace Elements of Anhydrous Minerals by LA-ICP-MS without Applying an Internal Standard, Chem. Geol. 257, 34 (2008).

[66] U. Schaltegger, A. K. Schmitt, and M. S. A. Horstwood, U-Th-Pb Zircon Geochronology by ID-TIMS, SIMS, and Laser Ablation ICP-MS: Recipes, Interpretations, and Opportunities, Chem. Geol. 402, 89 (2015).

[67] M. S. Dood, D. Papineau, T. Grenne, J. F. Slack, M. Rittner, F. Pirajno, J. O'Neil, and C. T. S. Little, Evidence for Early Life in Earth's Oldest Hydrothermal Vent Precipitates, Nature (London) 543, 60 (2017).

[68] J. H. M. Neijzen and A. Donszelmann, Hyperfine Structure and Isotope Shift Measurements in Neutral Gallium and Indium with a Pulsed Dye Laser, Physica (Amsterdam) 98C, 235 (1980).

[69] W. Nörtershäuser et al., Nuclear Charge Radii of ${ }^{7,9,10} \mathrm{Be}$ and the One-Neutron Halo Nucleus ${ }^{11} \mathrm{Be}$, Phys. Rev. Lett. 102, 062503 (2009).

[70] B. K. Sahoo, Accurate Estimate of $\alpha$ Variatin and Isotope Shift Parameters in $\mathrm{Na}$ and $\mathrm{Mg}^{+}$, J. Phys. B 43, 231001 (2010).

[71] M. R. Kalita et al., Isotope Shifts in the $7 s \rightarrow 8 s$ Transition of Francium: Measurements and Comparison to Ab Initio Theory, Phys. Rev. A 97, 042507 (2018).

[72] C. Delaunay, R. Ozeri, G. Perez, and Y. Soreq, Probing Atomic Higgs-like Forces at the Precision Frontier, Phys. Rev. D 96, 093001 (2017).

[73] V. V. Flambaum, A. J. Geddes, and A. V. Viatkina, Isotope Shift, Nonlinearity of King Plots, and the Search for New Particles, Phys. Rev. A 97, 032510 (2018).

[74] B. K. Sahoo, G. Gopakumar, R. K. Chaudhuri, B. P. Das, H. Merlitz, U. S. Mahapatra, and D. Mukherjee, Magnetic Dipole Hyperfine Interactions in ${ }^{137} \mathrm{Ba}^{+}$and the Accuracies of the Neutral Weak Interaction Matrix Elements, Phys. Rev. A 68, 040501(R) (2003).

[75] J. R. Persson, Table of Hyperfine Anomaly in Atomic Systems, At. Data Nucl. Data Tables 99, 62 (2013).

[76] C. J. Batty, S. F. Biagi, R. A. J. Riddle, B. L. Roberts, G. J. Pyle, G. T. A. Squier, D. M. Asbury, and A. S. Clough, Nuclear Quadrupole Deformation Effects on Pionic and Kaonic X-Rays, Nucl. Phys. A355, 383 (1981).

[77] J. N. P. Van Stralen and L. Visscher, The Nuclear Quadrupole Moment of ${ }^{115}$ In from Molecular Data, J. Chem. Phys. 117, 3103 (2002).

[78] T. G. Eck, A. Lurio, and P. Kusch, Hfs of the $5^{2} P_{\frac{1}{2}}$ State of ${ }^{115} \mathrm{In}$ and ${ }^{113} \mathrm{In}$ : Hfs Anomalies in the Stable Isotopes of Indium, Phys. Rev. 106, 954 (1957).

[79] B. M. Patterson, J. F. Sell, T. Ehrenreich, M. A. Gearba, G. M. Brooke, J. Scoville, and R. J. Knize, Lifetime Measurement of the Cesium ${ }^{6} P_{3 / 2}$ Level Using Ultrafast Pump-Probe Laser Pulses, Phys. Rev. A 91, 012506 (2015).

[80] S. Elhatisari, D. Lee, G. Rupak, E. Epelbaum, H. Krebs, T. A. Lähde, T. Luu, and U.-G. Meißner, Ab Initio AlphaAlpha Scattering, Nature (London) 528, 111 (2015).

[81] R. Kanungo et al., Proton Distribution Radii of ${ }^{12-19} \mathrm{C}$ Illuminate Features of Neutron Halos, Phys. Rev. Lett. 117, 102501 (2016). 
[82] A. Kumar et al., Nuclear Force Imprints Revealed on the Elastic Scattering of Protons with ${ }^{10} \mathrm{C}$, Phys. Rev. Lett. 118, 262502 (2017).

[83] B. J. Wood and A. N. Halliday, The Lead Isotopic Age of the Earth Can be Explained by Core Formation Alone, Nature (London) 465, 767 (2010).

[84] R. E. March, An Introduction to Quadrupole Ion Trap Mass Spectrometry, J. Mass Spectrom. 32, 351 (1997).

[85] T. Brunner et al., TITAN's Digital RFQ Ion Beam Cooler and Buncher, Operation and Performance, Nucl. Instrum. Methods Phys. Res., Sect. A 676, 32 (2012).

[86] E. S. Shuman, J. F. Barry, and D. DeMille, Laser Cooling of a Diatomic Molecule, Nature (London) 467, 820 (2010).

[87] J. F. Barry et al., Magneto-optical Trapping of a Diatomic Molecule, Nature (London)512, 286 (2014).

[88] T. A. Isaev and R. Berger, Polyatomic Candidates for Cooling of Molecules with Lasers from Simple Theoretical Concepts, Phys. Rev. Lett. 116, 063006 (2016).

[89] D. DeMille, J. M. Doyle, and A. O. Sushkov, Probing the Frontiers of Particle Physics with Tabletop-Scale Experiments, Science 357, 990 (2017).

[90] R. F. Garcia Ruiz et al., Collinear Resonance Ionization Spectroscopy of RaF Molecules, Report No. CERN-INTC2018-P546, 2018.

[91] J. Aldegunde and J. M. Hutson, Hyperfine Structure of ${ }^{2} \Sigma$ Molecules Containing Alkaline-Earth-Metal Atoms, Phys. Rev. A 97, 042505 (2018).

[92] I. Kozyryev and N. R. Hutzler, Precision Measurement of Time-Reversal Symmetry Violation with Laser-Cooled Polyatomic Molecules, Phys. Rev. Lett. 119, 133002 (2017).

[93] N. Kang-Kuen, H. Loh, M. Grau, K. C. Cossel, J. Ye, and E. A. Cornell, State-Specific Detection of Trapped $\mathrm{HfF}^{+}$by Photodissociation, J. Mol. Spectrosc. 300, 12 (2014).

[94] Y. A. Kudriavtsev and V. S. Letokhov, Laser Method of Highly Selective Detection of Rare Radioactive Isotopes through Multistep Photoionization of Accelerated Atoms, Appl. Phys. B 29, 219 (1982).

[95] D. Hanstorp, A Secondary Emission Detector Capable of Preventing Detection of the Photoelectric Effect Induced by Pulsed Lasers., Meas. Sci. Technol. 3, 523 (1992).
[96] S. McLuckey and J. M. Wells, Mass Analysis at the Advent of the 21st Century, Chem. Rev. 101, 571 (2001).

[97] T. Næraa, A. Scherstén, M. T. Rosing, A. I. S. Kemp, J. E. Hoffmann, T. F. Kokfelt, and M. J. Whitehouse, Hafnium Isotope Evidence for a Transition in the Dynamics of Continental Growth 3.2 Gyr Ago, Nature (London) 485, 627 (2012).

[98] Z. Wang and B. Harry, Ratios of S, Se and Te in the Silicate Earth Require a Volatile-Rich Late Veneer, Nature (London) 499, 328 (2013).

[99] S. Maher, F. P. M. Jjunju, and S. Taylor, Colloquium: 100 Years of Mass Spectrometry: Perspectives and Future Trends, Rev. Mod. Phys. 87, 113 (2015).

[100] R. Stoian, A. Rosenfeld, D. Ashkenasi, I. V. Hertel, N. M. Bulgakova, and E. E. B. Campbell, Surface Charging and Impulsive Ion Ejection during Ultrashort Pulsed Laser Ablation, Phys. Rev. Lett. 88, 097603 (2002).

[101] B. M. Hegelich, B. J. Albright, J. Cobble, K. Flippo, S. Letzring, M. Paffett, H. Ruhl, J. Schreiber, R. K. Schulze, and J. C. Fernández, Laser Acceleration of Quasi-Monoenergetic MeV Ion Beams, Nature (London) 439, 441 (2006).

[102] A. Macchi, M. Borghesi, and M. Passoni, Ion Acceleration by Superintense Laser-Plasma Interaction, Rev. Mod. Phys. 85, 751 (2013).

[103] M. Hada et al., Cold Ablation Driven by Localized Forces in Alkali Halides, Nat. Commun. 5, 3863 (2014).

[104] A. M. Elsied, N.C. Termini, P. K. Diwakar, and A. Hassanein, Characteristics of Ions Emission from Ultrashort Laser Produced Plasma, Sci. Rep. 6, 38256 (2016).

[105] S. Amoruso, R. Bruzzese, N. Spinelli, and R. Velotta, Characterization of Laser-Ablation Plasmas, J. Phys. B 32, R131 (1999).

[106] R. Russo, X. Mao, J. J. Gonzalez, V. Zorba, and J. Yoo, Laser Ablation in Analytical Chemistry, Anal. Chem. 85, 6162 (2013).

[107] C. Kerse et al., Ablation-Cooled Material Removal with Ultrafast Bursts of Pulses, Nature (London) 537, 84 (2016). 